University of South Florida

DIGITAL COMMONS

Digital Commons @ University of

@ UNIVERSITY OF SOUTH FLORIDA

South Florida

USF Tampa Graduate Theses and Dissertations

USF Graduate Theses and Dissertations

3-29-2006

\title{
Association of Sound to Motion in Video Using Perceptual Organization
}

Sunil Babu Ravulapalli

University of South Florida

Follow this and additional works at: https://digitalcommons.usf.edu/etd

Part of the American Studies Commons, and the Computer Engineering Commons

\section{Scholar Commons Citation}

Ravulapalli, Sunil Babu, "Association of Sound to Motion in Video Using Perceptual Organization" (2006). USF Tampa Graduate Theses and Dissertations.

https://digitalcommons.usf.edu/etd/3769

This Thesis is brought to you for free and open access by the USF Graduate Theses and Dissertations at Digital Commons @ University of South Florida. It has been accepted for inclusion in USF Tampa Graduate Theses and Dissertations by an authorized administrator of Digital Commons @ University of South Florida. For more information, please contact digitalcommons@usf.edu. 
Association of Sound to Motion in Video Using Perceptual Organization

by

Sunil Babu Ravulapalli

A thesis submitted in partial fulfillment

of the requirements for the degree of

Master of Science in Computer Science and Engineering

Department of Computer Science and Engineering

College of Engineering

University of South Florida

Major Professor: S. Sarkar, Ph.D.

Rangachar Kasturi, Ph.D.

Ravi Sankar, Ph.D.

Date of Approval:

March 29, 2006

Keywords: Video Surveillance, Sound Association, Auditory Scene Analysis, Auditory Object, Motion Detection

(C) Copyright 2006, Sunil Babu Ravulapalli 


\section{ACKNOWLEDGEMENTS}

I would like to thank Dr. Sudeep Sarkar for giving me this opportunity to work with him. I am grateful to him for his enthusiasm for research and moral support. Without his patient guidance this work would not have been possible. I also thank Dr. Rangachari Kasturi \& Dr. Ravi Sankar for the valuable time they took to review this thesis and their helpful comments.

I am very grateful to my loving Father, Mother, and Sister without whose moral support this work would not have reached the completion. I am indebted to them for being a perpetual source of inspiration and motivation for me. Finally, I cannot forget the help and support of all my friends who have been with me in every step of this process. 


\section{TABLE OF CONTENTS}

LIST OF TABLES $\quad$ ii

LIST OF FIGURES $\quad$ iii

ABSTRACT vi

CHAPTER 1 INTRODUCTION 1

1.1 Cross Modality in Human Perception 2

1.2 Cross Modality in Artificial Perception 6

1.3 Contribution of this Thesis 8

1.4 Layout of Thesis 8

CHAPTER 2 HUMAN PERCEPTION OF SOUND 9

2.1 Cues Used by ASA Process 9

2.2 Effects of ASA on Perception 10

2.3 Similarity in Audio and Vision 11

2.4 Theory of Indispensable Attributes 11

CHAPTER 3 AUDIO VIDEO ASSOCIATION 14

$\begin{array}{lll}3.1 & \text { Grouping Sound Events } & 14\end{array}$

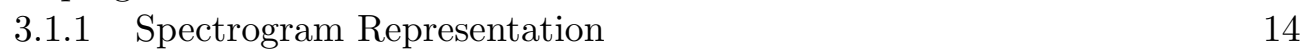

$\begin{array}{lll}3.1 .2 & \text { Line Detection } & 18\end{array}$

$\begin{array}{lll}3.1 .3 & \text { Grouping } & 20\end{array}$

3.1.4 Property Extraction 20

3.2 Grouping Video Objects 21

3.3 Association 22

CHAPTER 4 RESULTS 26

4.1 Scenario 1: Person Bouncing Ball and Walking Person 26

4.2 Scenario 2: Walking Person and Moving Car 28

4.3 Scenario 3: Person Walking and Another Person Running 29

4.4 Scenario 4: Person Walking and Bouncing Ball 30

4.5 Scenario 5: Person Walking, Person Running and Bouncing Ball 31

$\begin{array}{lll}\text { CHAPTER } 5 & \text { CONCLUSIONS } & 36\end{array}$

$\begin{array}{ll}\text { REFERENCES } & 37\end{array}$ 


\section{LIST OF TABLES}

$\begin{array}{lll}\text { Table } 3.1 & \text { Orientation Groups } & 20\end{array}$

Table 4.1 Difference Percentages: Video Object $1=$ Bouncing Ball Video Object $2=$ Footsteps 


\section{LIST OF FIGURES}

Figure 1.1 Simplified Representation of a Stimulus Sequence that Exhibits

"Freezing" Effect [5] 3

Figure 1.2 Simplified Representation of a Stimulus to Measure Motion After Effect (MAE) [6]

Figure 1.3 Attentional Modulation of MAE Perception [6] 5

Figure 1.4 Attentional Modulation of fMRI Decay Time in Area MT+ 6$] \quad 6$

Figure 2.1 Grouping by Gestalt Principles (a) Grouping by Proximity (b) Grouping by Similarity [2]

Figure 2.2 An Illusion Which is Seen as Two Faces or a Vase Depending on Our Interpretaion of the Background

Figure 2.3 Theory of Indispensable Attributes in Vision (a) Two Yellow Spots at Same Spot, Observer Sees One Yellow Spot (b) One Yellow and One Blue Create One White Spot (c) Two Lights Create Two Separate Spots, Regardless of Color Observer Sees Two Spots [18]

Figure 2.4 Theory of Indispensable Attributes in Audio (a) One Speaker Plays Two Ds, Listener Hears One D Sound (b) One Speaker Plays D While Another Plays a D, Listener Hears One Sound (c) D and F are Played Over One Speaker, Listener Hears Two Sounds [18]

Figure 3.1 Overview of Association of Sound to Object in Video

Figure 3.2 Computational Model of Grouping Sound Events

Figure 3.3 Spectrograms (Top Left) Footsteps and Moving Car (Top Right) Chirping Bird and Car Horn (Bottom Left) Car Horn (Bottom Right) Bouncing Ball and Footsteps

Figure 3.4 Thresholded Spectrograms (Top Left) Footsteps and Moving Car (Top Right) Sound of Chirping Bird and Car Horn (Bottom Left) Car Horn (Bottom Right) Bouncing Ball and Footsteps 
Figure 3.5 Spectrogram Images with Detected Lines (Top Left) Footsteps and Moving Car (Top Right) Chirping Bird and Car Horn (Bottom Left) Car Horn (Bottom Right) Bouncing Ball and Footsteps

Figure 3.6 Isolated Auditory Events (Top Left) Bird Chirping (Top Right) Footsteps (Middle Left) Ball Bounce (Middle Right) Footsteps (Bottom Left) Car Horn (Bottom Right) Truck Horn

Figure 3.7 Column Wise Summation of an Auditory Group 24

Figure 3.8 A Walking Person Exhibits Shape Change Periodicity 24

Figure 3.9 A Bouncing Ball Exhibits Track Change Periodicity 25

Figure 3.10 (Top) Spectrogram of the Sound Waveform Received. (Bottom) Corresponding Frames for Each Event in the Spectrogram

Figure 4.1 (Left) Spectrogram (Middle) Thresholded Spectrogram (Right) Detected Lines

Figure 4.2 Audio Groups

Figure 4.3 (Top) Video Frames (Middle) Thresholded Frames (Bottom) Periodicity Curves

Figure 4.4 Association of Sound - Top Row Shows the Lines Detected from the Spectrogram of the Sound Wave from Which We Obtain Audio Objects Shown in the Second Row. Third Row Shows the Periodicity Curves of Objects in the Video Extracted from Video Frames Shown in the Last Row

Figure 4.5 Spectrogram and the Corresponding Audio Groups 30

$\begin{array}{lll}\text { Figure } 4.6 & \text { (Top) Video Frames (Bottom) Periodicity Curves } & 31\end{array}$

Figure 4.7 Top Row Shows the Spectrogram on the Left Side and Lines Detected on the Right Side. Two of the Audio Objects are Shown in the Third Row. Fourth Row Shows the Periodicity Curves of Objects in the Video Extracted from Video Frames Shown in the Last Row

Figure 4.8 Top Three Rows Show the Spectrogram, Detected Lines and the Audio Objects. Fourth Row Shows the Periodicity Curves of Objects in the Video. The Fifth Row Shows Thresholded Frames and the Last Row Shows Video Frames

Figure 4.9 Top Row Shows the Spectrogram and the Next Row Shows the Lines Detected. Two of the Audio Objects are Shown in the Third Row. Fourth Row Shows the Periodicity Curves of Objects in the Video Extracted from Video Frames Shown in the Last Row 
Figure 4.10 Top Row Shows the Lines from the Spectrogram. Three of the Audio Objects are Shown in the Second Row. Third Row Shows the Periodicity Curves of Objects in the Video Extracted from Video Frames Shown in the Last Row 


\title{
ASSOCIATION OF SOUND TO MOTION IN VIDEO USING PERCEPTUAL ORGANIZATION
}

\author{
Sunil Babu Ravulapalli
}

\begin{abstract}
Technological developments and innovations of the first forty years of the digital era have primarily addressed either the audio or the visual senses. Consequently, designers have primarily focused on the audio or the visual aspects of design. In the perspective of video surveillance, the data under consideration has always been visual. However, in light of the new behavioral and physiological studies which established a proof of cross modality in human perception i.e. humans do not process audio and visual stimulus separately, but percieve a scene based on all stimulus available, similar cues are being used to develop a surveillance system which uses both audio and visual data available. Human beings can easily associate a particular sound to an object in the surrounding. Drawing from such studies, we demonstrate a technique by which we can isolate concurrent audio and video events and associate them based on perceptual grouping principles. Associating sound to an object can form a part of larger surveillance system by producing a better description of objects.

We represent audio in the pitch-time domain and use image processing algorithms such as line detection to isolate significant events. These events and are then grouped based on gestalt principles of proximity and similarity which operates in audio. Once auditory events are isolated we can extract their periodicity. In video, we can extract objects by using simple background subtraction. We extract motion and shape periodicities of all the objects by tracking their position or the number of pixels in each frame. By comparing all the periodicities in audio and video using a simple index we can easily associate audio to video.
\end{abstract}


We show results on five scenarios in outdoor settings with different kinds of human activity such as running, walking and other moving objects such as balls and cars. 


\section{CHAPTER 1 INTRODUCTION}

Automated surveillance addresses real-time observation of people, vehicles and other moving objects within a complicated environment, leading to a description of their actions and interactions. The technical issues include moving object detection and tracking, object classification, human motion analysis and activity understanding. Most commonly used sensors for surveillance are imaging sensors, e.g. video cameras and thermal imaging systems. There are a number of video surveillance systems based on a single camera or hundreds of cameras. To achieve continuous monitoring, infrared (IR) cameras are used along with the optical cameras under low illuminations. However, motion detection and tracking are often uncertain and incomplete as they require recognition methods that can handle the probabilities accurately. Off late "detection and tracking" systems based on non-imaging measurements are being experimented with. Measurement of force on load cells [1] has already been used. Though the performance of such sensors is high, the cost of their usage is also very high. In this regard the microphone turns our to be a good sensor due its compact size, low cost, small data volume and low power consumption. Also, recent experiments in the psychological domain have shown that human perception does not process senses such as visual, sound, smell separately but rather perceives a scene based on the fusion of all the modalities available at a particular instant. This has prompted researchers in the computer vision community to make use of the rich multimedia information (especially audio) in a video sequence for video surveillance. Several studies on fusing audio-video data for object detection and tracking have been reported in the literature. In the following section we will take a look at human perception which formed the basis for the use of cross-modal fusion in artificial systems. Then we go on to look at some literature that use cross-modal fusion in artificial systems. 


\subsection{Cross Modality in Human Perception}

Bergman in [2] described a number of Gestalt principles for auditory scene analysis in which he stressed the resemblance between audition and vision, because principles of perceptual organisation such as similarity, good continuation, common fate seem to play a similar role in the two modalities. This similarity led researchers to believe that a human perceptual system might utilize information from all available modalities, thus giving rise to interest in the cross-modal domain.

There are several examples of cross modal influences in which one may assume that perceptual system indeed contributes information from different sensory modalities to an single event. There is quite a bit of literature showing that arbitrary combinations of modalities heighten perceptual awareness and lower reaction time compared with just the unimodal features. Cross-modal combinations of features not only enhance perceptual processing but can also change percept. The prime example is the McGurk effect [3], in which speech information from sound and vision is presented. When listeners hear "baba" and at the same time watch a speaker articulating "gaga", they tend to combine the information from the two sources into "dada". Cross modal interactions have also been observed in spatial domain. For example synchronized sounds and light flashes with a different spatial location tend to be localized closer together (the ventriloquist effect). The common finding is that there is a substantial effect of the light flashes on the location of the sound [4].

In a series of experiments Vroomen and De Gelder explored something they called "freezing effect" [5]. It is an illusion that occurs when an abrupt sound is presented during a rapidly changing visual display. In this illusion the participant feels as if the sound is pinning the visual stimulus for a short moment so that the visual display "freezes" helping the participant to identify the visual. Participants saw a $4 \times 4$ matrix of flickering dots that was created by rapidly presenting four different displays alternated by a mask (Figure 1.1). Each visual contained four dots in random positions. One of the four displays consisted of four dots that made up a diamond, and it could appear in any four corners of the matrix. The task of the participants was to detect the position of the diamond. One set of experiments involved playing only low tones being played along with all the visuals, in another experiment a high 


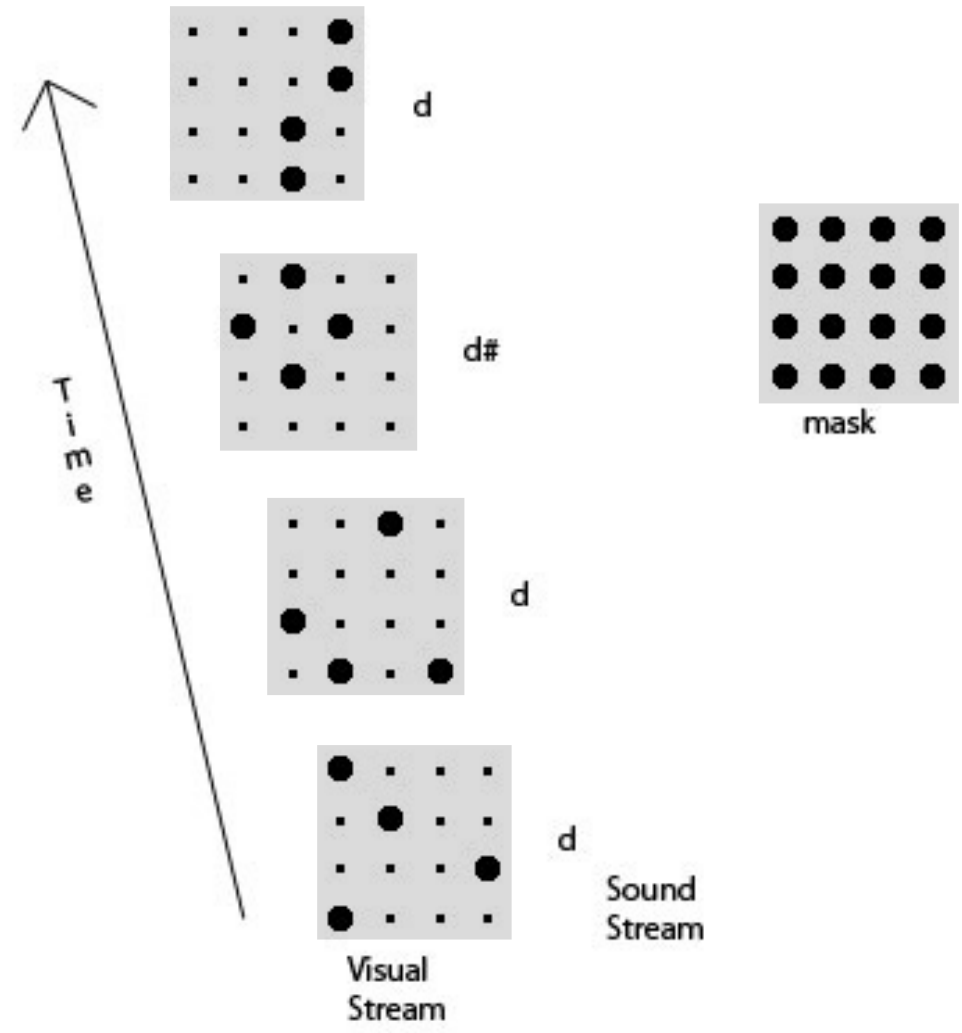

Figure 1.1 Simplified Representation of a Stimulus Sequence that Exhibits "Freezing" Effect $[5]$

tone was played along with the target visual. The average number correct responses was $55 \%$ when only low tones were played and $66 \%$ when a high tone was played along with the target.

In another experiment psychophysical and functional magnetic resonance imaging (fMRI) data was acquired while subjects processed a given situation. A visual illusion which produces motion aftereffect (MAE) was used to assess the effects of visual and auditory attention on motion processing in the human area MT+ (dorsal stream visual area). The MAE occurs after a period of motion adaption, in which subjects view a unidirectionally moving stimulus. When subjects subsequently view a stationary stimulus, it appears to move in the opposite direction for a short period of time [6]. Subjects performed three scans in a single imaging session [7]: 


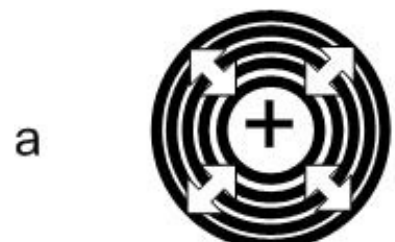

Reversing motion

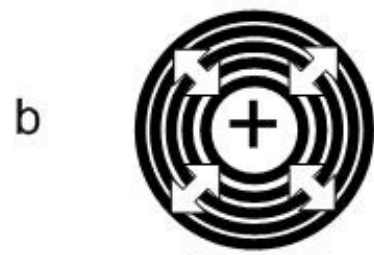

Reversing motion

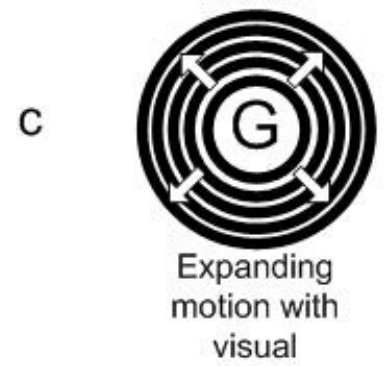

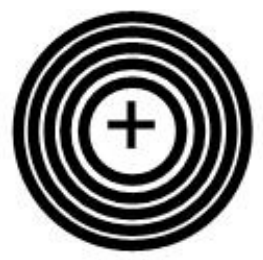

static

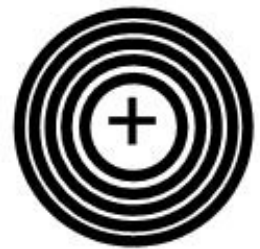

static

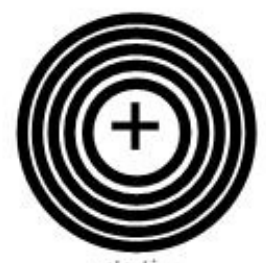

static

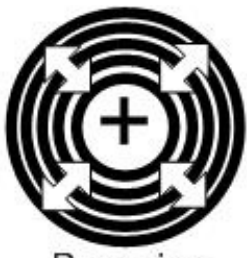

Reversing

motion

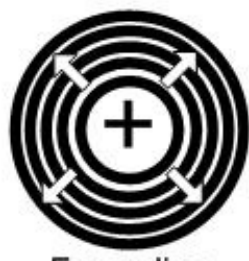

Expanding

motion

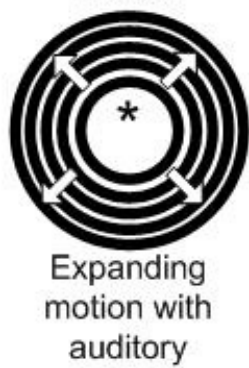

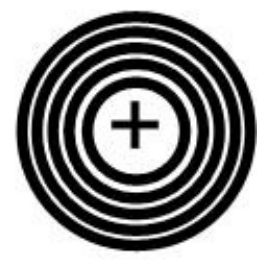

static

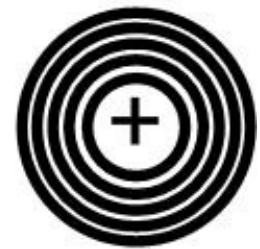

static

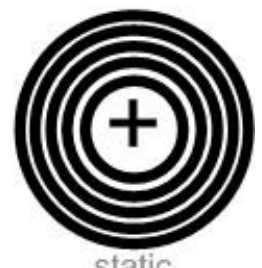

static

Figure 1.2 Simplified Representation of a Stimulus to Measure Motion After Effect (MAE) [6]

- Localizer scan

- Fixation-only scan

- Attentional MAE scan

The localizer scan (Figure 1.2a) was used to identify area MT+ in each subject. In this scan subjects fixated a central crosshair and passively viewed reversing stimuli alternated by a stationary stimuli. This stimulus however does not induce any MAE. The fixation-only scan (Figure 1.2b) was designed to measure MT+ activity and the MAE in the absence of attentional demands. Blocks of moving and stationary stimuli were alternated. In half of the moving blocks, the stimulus moved only in the outward direction so as to induce motion aftereffect. The attentional MAE scan (Figure 1.2c) was designed to determine whether attention to a central visual or auditory stimulus during motion adaption has an impact on signal in area MT+ and the MAE. In all of the moving blocks, the stimulus moved only in the 


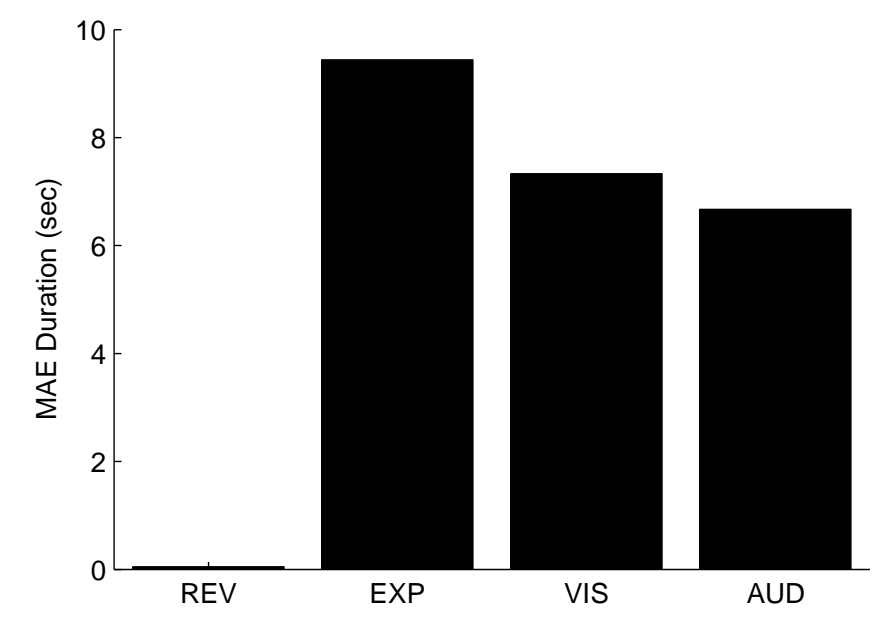

Figure 1.3 Attentional Modulation of MAE Perception [6]

outward direction. Subjects performed either a visual or an auditory task during the moving block. In the visual task, subjects saw a series of letters in random order, which replaced the fixation crosshair. In the auditory task, subjects heard a series of letters presented at the same rate as the moving blocks. For both visual and auditory tasks, subjects were instructed to press a button whenever they saw or heard a vowel. Also, functional and structural MR data were obtained using a 1.5 T GE magnet and custom-designed head coil.

Duration of the MAE after expanding motion, averaged across subjects, was 9.44 seconds (Figure 1.3). MAE duration was reduced when subjects attended to the central visual task during expanding motion, to an average of 7.54 seconds. Also when subjects attended to an auditory task during expanding motion, duration of MAE was reduced to 6.67 seconds.

Similarly, the signal in MT+ remained higher after blocks that induced a motion aftereffect than after blocks when no aftereffect accured. This is shown by the markedly prolonged decay time for the expanding motion condition as compared to the reversing motion condition (Figure 1.4). The decay time following blocks of expanding motion was reduced significantly when subjects attended to the central visual task during adaption. It was also found that auditory attention alters the decay time in area MT+. These results demonstrate that auditory attention can influence motion processing in an early visual area. 


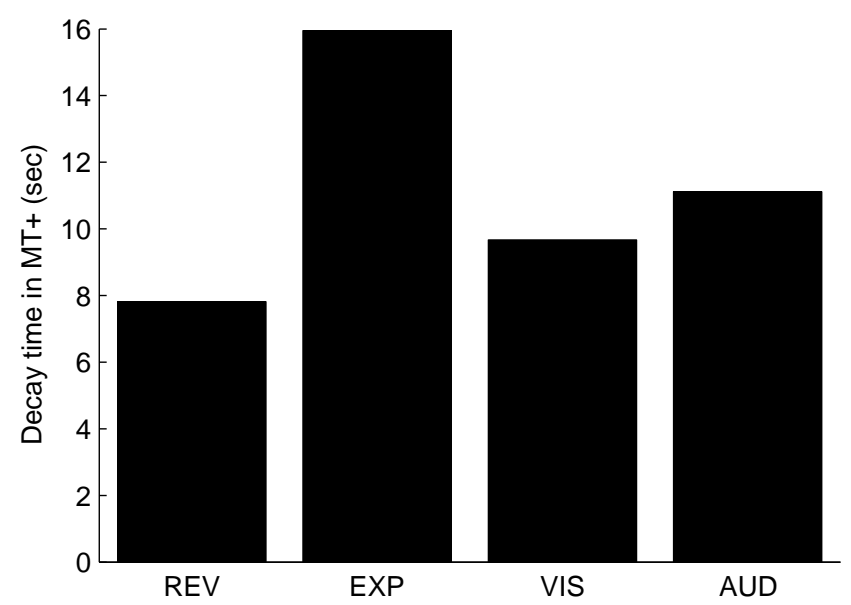

Figure 1.4 Attentional Modulation of fMRI Decay Time in Area MT+ [6]

\subsection{Cross Modality in Artificial Perception}

A greater understanding of the human perception has led reseachers to use cross modality in numerous projects for increase in accuracy and reliability. The use of cross modality has already appeared in localization in video, surveillance and multimedia indexing. Lo and Goubran proposed a new method for performing audio-video talker localization [8] that explores the reliability of the individual localization estimates such as audio, motion detection, and skin color detection. Audio signals are captured by a six element microphone array. The talkers direction is obtained through a beamforming algorithm. Audio data is digitized and localization is performed using the delay-and-sum-method [9]. In video, motion detection is done using background subtraction and for skin color detection the luma-chroma space is used. The reliability information is estimated from the audio and video separately. The reliability information in conjunction with a simple summing voter is used to fuse the localization results. Based on the voter output, a majority rule is then used to make final decision of the active talkers current location. The results show that adding the reliability information during fusion improves localization performance when compared to audio only, motion detection only or skin color detection only.

Lately surveillance systems are using both audio and video sensors to reveal and track the presence of an intruder. The system described in [10] is composed of a mobile agent and several 
static agents cooperating in the tracking task. The mobile agent is a vision agent composed of an omni-directional vision system and a mobile robot. The static agents are acoustic agents composed of self steerable microphone arrays. To detect the intruder the image is segmented into the moving foreground and into the static background. An incremental background subtraction algorithm used to account for a dynamic environment. In this technique the backgorund image is not a static image, but is updated frame after frame slowly incorporating changes in the scene. The acoustic agent receives the position of the intruder from the static agent, a beamforming algorithm is used to direct the microphone array toward the acoustic source. As a sequence of frame is obtained, the signal is reconstructed using the overlapadd method to the result of the IFFT block. The acoustic signal obtained is used to train a HMM (Hidden Markov Model) of the steps of the intruder. When the person moves, the learnt HMM can be used to distinguish one person moving in the environment from another one. The acoustic agent is also able to calculate the position of the intruder with respect to itself. The localisation algorithm is implemented using a neural network based algorithm. The measurements on the position of the intruder coming from the static acoustic agents are fused using a modified Kalman filter.

In [11] a content based video parsing and indexing method is presented which analyzes both information sources (audio and video) and accounts for their inter-relations and synergy to extract high-level semantic information. Low-level audio analysis involves the tasks of speech-silence discrimination, linear predictive (LP) analysis and derivation of LP cepstral coefficients of the speech-voiced frames. The visual input is the entire video sequence on which a shot boundary (scene-cut) detection and video segmentation is performed. The first frame of each detected shot is subsequently processed by a face detection algorithm followed by a facial feature extraction module aiming at extracting mouth location. A mouth template is thus obtained and used for mouth tracking in the remaining frames of the face shot. Multimodal interaction can serve to enhance the content findings and offer a more detailed content description about the same video instances. For example, since speaker recognition performed on the audio source is prone to recognition errors, visual information can be used to detect presence of talking people and the speaker that exhibits maximum presence likelihood is the winner. The interaction of audio and visual semantic labels such as speech, silence, speaker 
identity, face presence, face absence supplies the user with more detailed information and a degree of underlying context.

Speaker localisation using audio-video cues at signal level has been explored in [12]. It uses the correlation between the visual motion of the mouth and the corresponding audio data generated when a person speaks. A time delayed neural network is used to learn the audiovideo correlation which is then used to do a spatio-temporal search for a speaking person.

Kidron, Schechner and Elad present an method which detects pixels that are associated to a sound while filtering out other dynamic pixels [13]. They use canonical correlation analysis (CCA) by exploiting typical spatial sparsity of audio-visual events.

\subsection{Contribution of this Thesis}

The main contribution of this thesis consist of,

- Separating more than one concurrent audio and video events using a feature based approach based on single audio and a single video sensor.

- Usage of higher level primitives and grouping making audio-video association more robust.

- Use of cluttered environment where more than one object exists and associates sound to the corresponding object at a particular instant.

- Use of auditory scene analysis (ASA) rather than the usual signal processing approaches to process sound.

\subsection{Layout of Thesis}

This chapter provided motivation and background for this work. The next chapter gives an introduction to human perception of sound. The third chapter describes our approach for the audio-video association. The fourth chapter consists of the results. The final chapter concludes this work and lists the scope for future work. 


\section{CHAPTER 2 \\ HUMAN PERCEPTION OF SOUND}

Humans use their sense of hearing to understand the properties of sound-producing events. Often, we are interested in a single stream of events, such as a violin playing, a person talking, or a car approaching. In a natural listening environment, however, the acoustic energy produced by each event sequence is mixed, at the listener's ears, with energy arising from other concurrent events. "Auditory scene analysis" (ASA) is a process in which the auditory system takes the mixture of sound that it derives from a complex natural environment and sorts it into packages of acoustic evidence in which each package probably has arisen from a single source of sound [2]. The performance of the human auditory system with regard to the ability to analyze the auditory environment, the localization of sound sources and perception of speech is striking. Even in complex acoustical scenarios or under adverse acoustical conditions the human system can separate and recognise auditory events very robustly. ASA provides answers to how the brain can build separate perceptual descriptions of sound-generating events despite the mixing of evidence. The first thing it does is to analyze the incoming array of sound into

a large number of frequency components. Then by putting together the right set of frequency components over time, a signal is recognized. From the point of view of theoretical signal processing it is striking that the auditory system can achieve all this with just two receivers, the left and the right ear. As a consequence, these properties of the human auditory system have been a motivation to simulate it by means of computer models.

\subsection{Cues Used by ASA Process}

- The perceptual segregation of sounds in a sequence depends upon differences in their frequencies, pitches, timbres, amplitudes, and locations, and upon sudden changes of 
these variables. Segregation also increases as the duration of silence between sounds in the same frequency range gets longer.

- The perceptual fusion of simultaneous components to form single perceived sounds depends on their onset and offset, frequency separation, regularity of spectral spacing, harmonic relations and parallel amplitude modulation.

- Different cues for stream segregation compete to control the grouping, and different cues have different strengths.

- Primitive grouping occurs even when the frequency and timing of the sequence is unpredictable.

- An increased biasing toward stream segregation builds up with longer exposure to sounds in the same frequency region.

- Stream segregation is context-dependent, involving the competition of alternative organizations.

\subsection{Effects of ASA on Perception}

- A change in perceptual grouping can alter the perception of rhythms, melodic patterns, and overlap of sounds.

- Patterns of sounds whose members are distributed into more than one perceptual stream are much harder to perceive than those wholly contained within a single stream.

- Perceptual organization can affect perceived loudness and spatial location.

- The rules of ASA try to prevent the crossing of streams in frequency, whether the acoustic material is a sequence of discrete tones or continuously gliding tones.

- Known principles of ASA can predict the camouflage of melodies and rhythms when interfering sounds are interspersed or mixed with a to-be-recognized sequence of sounds.

- The apparent continuity of sounds through masking noise depends on ASA principles. Stimuli include frequency glides, amplitude-varying tones, and narrow-band noises. 


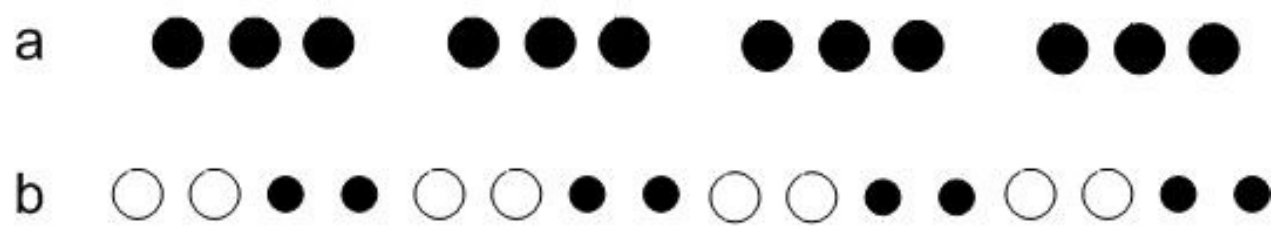

Figure 2.1 Grouping by Gestalt Principles (a) Grouping by Proximity (b) Grouping by Similarity [2]

- A perceptual stream can alter another one by capturing some of its elements.

- The apparent spatial position of a sound can be altered if some of its energy becomes grouped with other sounds.

- The segregation of streams of visual apparent motion works in exactly the same way as auditory stream segregation.

\subsection{Similarity in Audio and Vision}

Bergman [2] showed the similarities between the Gestalt principles in vision and audition. Just as grouping by proximity functions operates in visual space it also operates in auditory pitch. McPherson, Ciocca and Bergman [14] have shown that good continuation operates in audition in an analogous way to vision. The concept of amodal completion as it is used in vision [15] has been given a number of different names in audition: the acoustic tunnel [16] effect, perceptual restoration [17] and the continuity effect [2]. Since all these phenomenon abide by the same laws of grouping and organization, a framework that accounts for these needs to be used.

\subsection{Theory of Indispensable Attributes}

A perceptual object is that which is susceptible to figure-ground segregation. Early processing produces elements that require grouping. Grouping occurs following the principles described by the Gestalt psychologists (Figure 2.1); it produces Gestalts or perceptual organizations, which are also putative perceptual objects. Attention selects one putative object to become Figure 2.2 and relegates all othe information to ground [18]. There is little doubt 


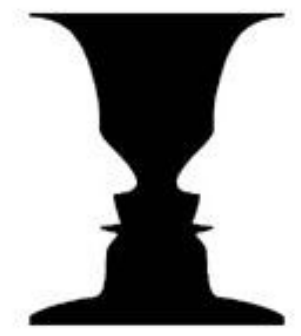

Figure 2.2 An Illusion Which is Seen as Two Faces or a Vase Depending on Our Interpretaion of the Background

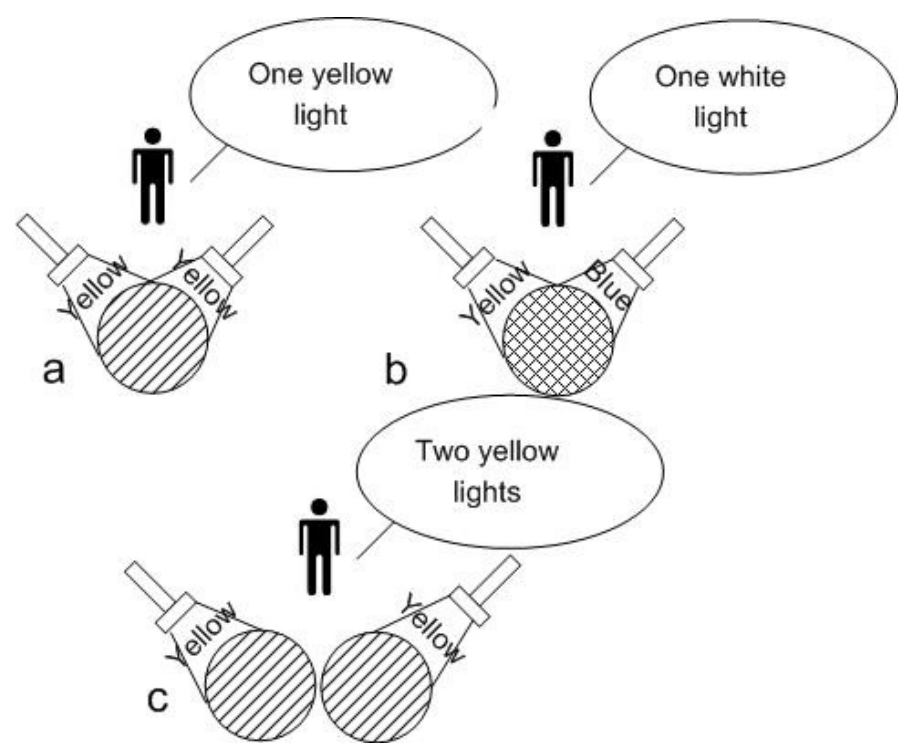

Figure 2.3 Theory of Indispensable Attributes in Vision (a) Two Yellow Spots at Same Spot, Observer Sees One Yellow Spot (b) One Yellow and One Blue Create One White Spot (c) Two Lights Create Two Separate Spots, Regardless of Color Observer Sees Two Spots [18]

that grouping and figure-ground segregation describe processes that are meaningful for auditory perception. Grouping is a well-established auditory phenomenon. Another phenomenon that characterizes visual objects is the formation and assignment of edges. However, edges in audio seems to be an abstract concept. Kubovy and Valkenburg [18] develop the theory of indispensable attributes (TIA) which states that, in vision, objects are formed in space-time domain, however auditory objects are formed in pitch-time domain. Spatial separation is an indespensible attribute for vision. Imagine presenting to an observer two spots of light on a surface (Figure 2.3a). Both of them yellow and they coincide; the observer will report one 


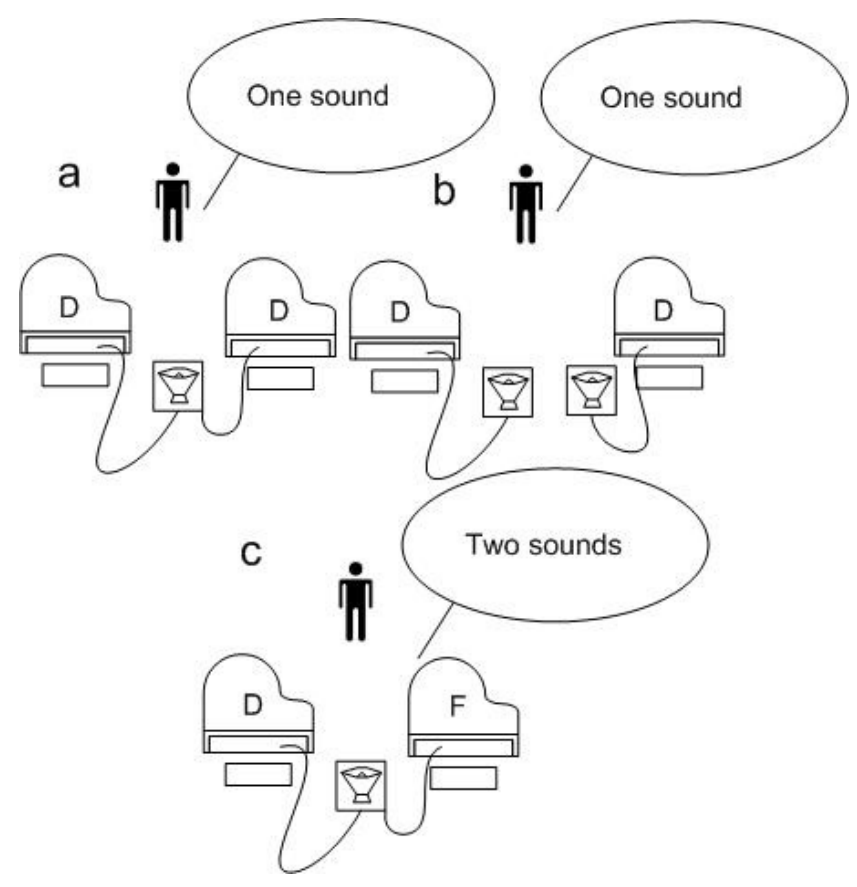

Figure 2.4 Theory of Indispensable Attributes in Audio (a) One Speaker Plays Two Ds, Listener Hears One D Sound (b) One Speaker Plays D While Another Plays a D, Listener Hears One Sound (c) D and F are Played Over One Speaker, Listener Hears Two Sounds [18]

light. Now suppose we change the color of the lights, so that one spot is blue and other is yellow, but they still coincide (Figure 2.3b); the observer will report one white light. For the observer to see more than one light, they must occupy different spatial location (Figure 2.3c).

Pitch separation is an indispensable attribute for sound. Imagine simultaneously playing two $440 \mathrm{~Hz}$ sounds for a listener (Figure 2.4a). Both of them played over the same loud speaker; the listener will report hearing one sound. Now suppose we play these two sounds over two loudspeakers; the listener will still report hearing one sound (Figure 2.4b). For the listener to report more than one sound, they must be separated in frequency. Thus, pitch separation is an indispensable attribute for audio perception. By analogous argument time is an indispensable attribute for both vision and audition. The TIA thus forms a heuristic tool for extending theories of visual perception into the domain of auditory perception. 


\section{CHAPTER 3}

\section{AUDIO VIDEO ASSOCIATION}

In this chapter we explain various audio and video algorithms used in this thesis. The overview of this technique is show in Fig. 3.1.

\subsection{Grouping Sound Events}

Traditionally, there have been several approches to audio signal processing. The signal processing approches to audio analysis are dominated by linear prediction and ceptrum analysis. Linear prediction is used for speech coding and ceptral features are the heart of speech recognition. Another popular technique, the Independent Component Analysis (ICA) tries to make the extracted sources as statistically independent as possible. However, ICA is a general purpose signal processing technique and requires as many mixtures as the number of sources. Also, it needs auditory specific constraints to be incorporated. Auditory Scene analysis (ASA) forms the next generation of audio processing. The overview of the steps used in this thesis to process audio is show in Figure 3.2.

\subsubsection{Spectrogram Representation}

As explained in the previous chapter, to process audio using ASA techniques we need a frame work in the pitch-time domain. In this thesis we use the spectrogram to represent audio. Spectrograms are usually calculated from the time signal using the Discrete-time Fourier transform (DTFT):

$$
X(w)=\sum_{n} x(n) e^{-j w n}
$$




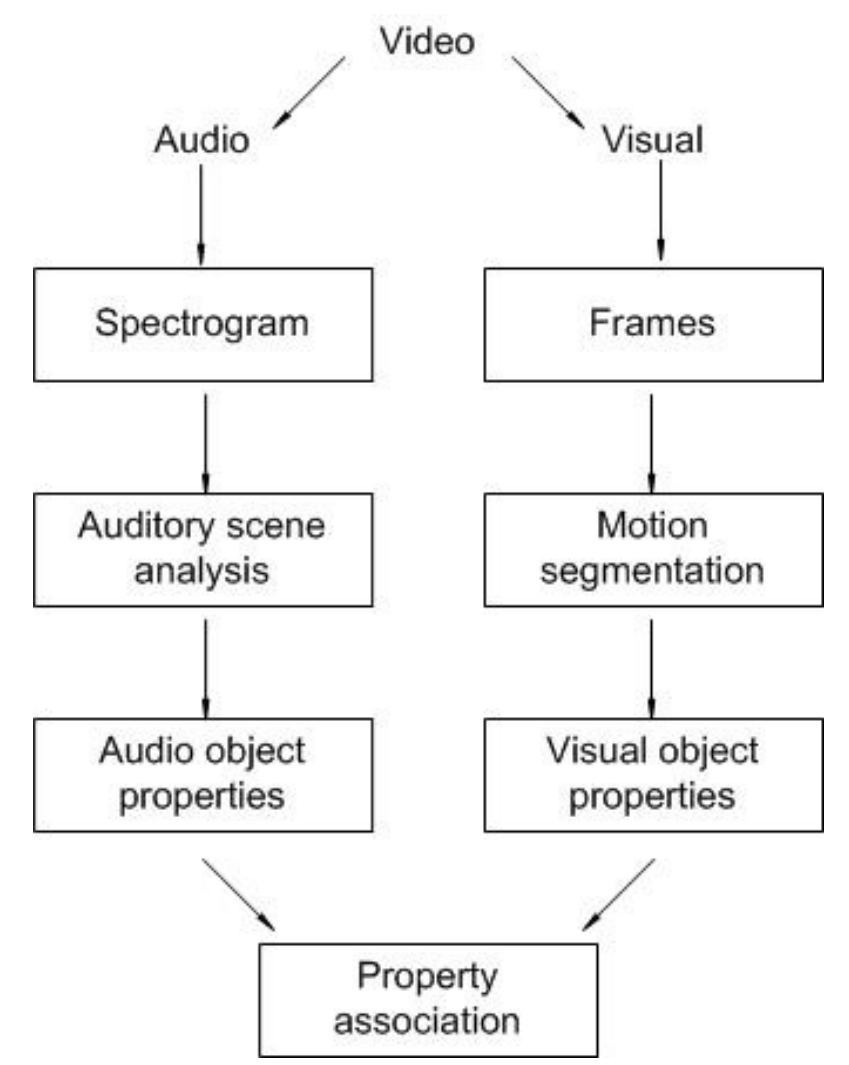

Figure 3.1 Overview of Association of Sound to Object in Video

If $x(n)$ is time-limited to a duration of $N$ samples, then we can sample the continuous function $X(w)$ at $N$ uniformly spaced points. This corresponds to forming a periodic signal $x^{\prime}(n)$ of infinite duration and period $N$ by concatenating the length $N$ sequences $x(n)$ and computing its Fourier Series expansion. This version of the Fourier Transform is written as:

$$
X(k)=\sum_{n=0}^{N} x(n) e^{-j w_{k} n}
$$

where $w_{k}=2 \pi k / N$

This expression is the Discrete Fourier Transform, or DFT, of the length $N$ discrete time sequence $x(n)$. Here, $k$ is the DFT bin number, and $w_{k}$ is the discrete frequency of bin $k$.

The spectrogram uses a slightly different form of DFT called the short time Fourier transform (STFT). The STFT is a formulation that can represent sequences of any length by breaking them into shorter blocks, or frames, and applying the DFT to each block. Digitally sampled data, in the time domain, is broken up into frames, which usually overlap, and Fourier 


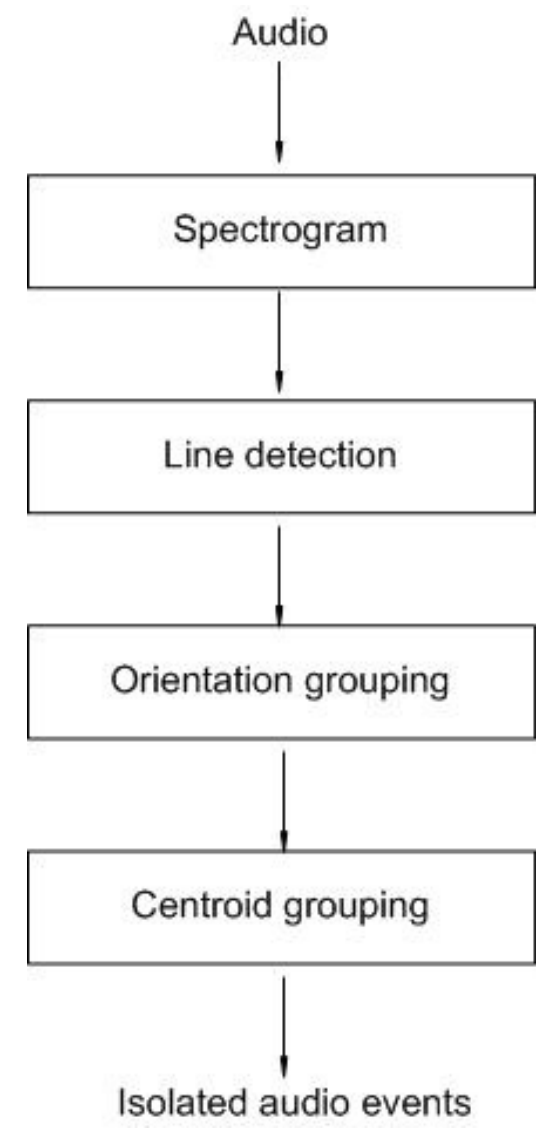

Figure 3.2 Computational Model of Grouping Sound Events

transformed to calculate the magnitude of the frequency spectrum for each frame. Each frame then corresponds to a vertical line in the image; a measurement of magnitude versus frequency for a specific moment in time. The spectrums or time plots are then 'laid side by side' to form the image. The horizontal axis represents time, the vertical axis is frequency, and the intensity of each point in the image represents amplitude of a particular frequency at a particular time.

$$
\operatorname{spectrogram}(t, w)=|\operatorname{STFT}(t, w)|^{2}
$$

There are several issues involved in choosing a good value for $N$. First, in order to take advantage of the computational efficiency of the FFT algorithm, we want to take $N$ to be a power of two. Secondly, the visual display produced by the analysis will be represented by $N$ samples of the DTFT. The larger we make $N$, the closer the DFT will approximate the smooth 

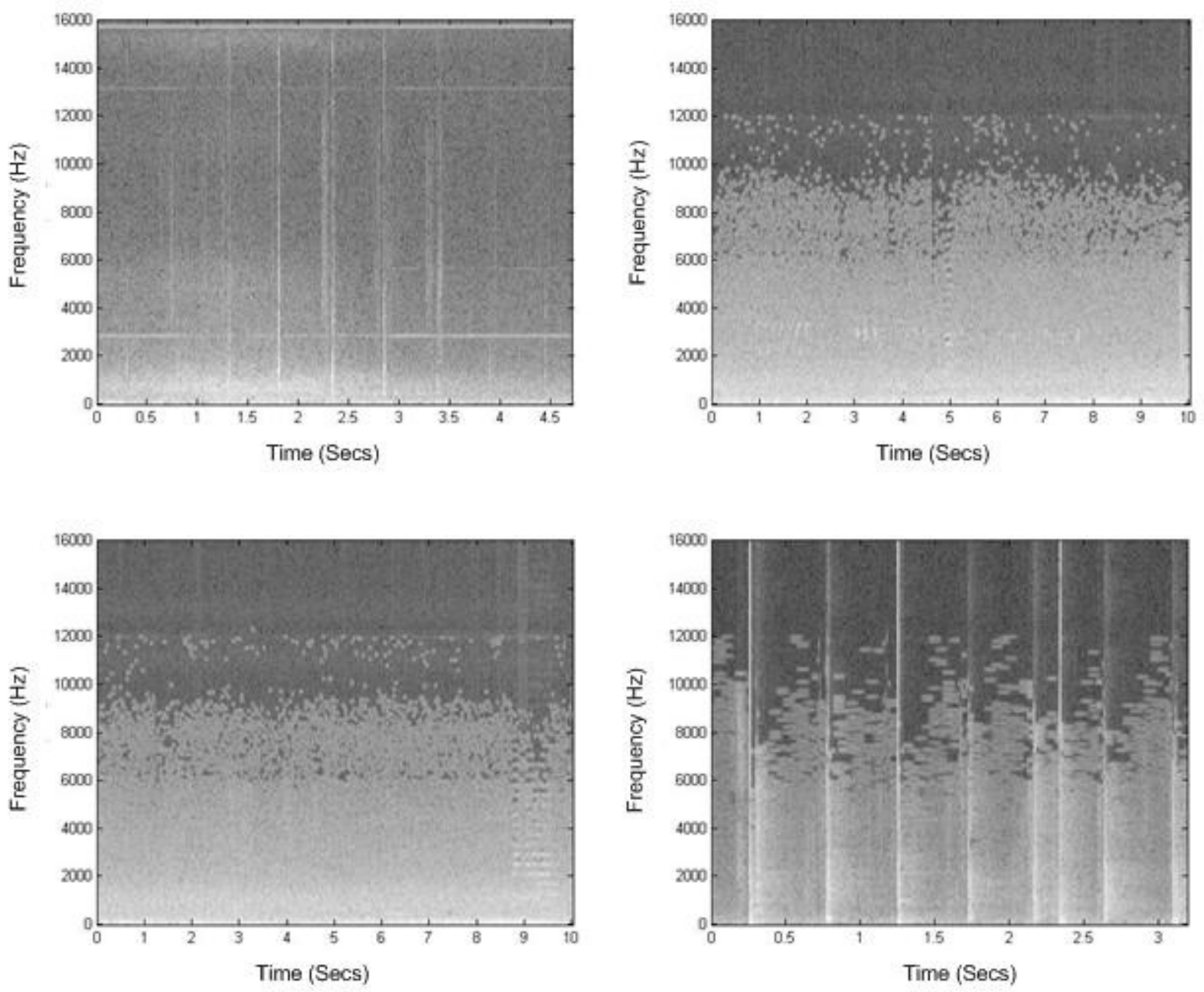

Figure 3.3 Spectrograms (Top Left) Footsteps and Moving Car (Top Right) Chirping Bird and Car Horn (Bottom Left) Car Horn (Bottom Right) Bouncing Ball and Footsteps

function represented by the DTFT. A value that is too small, while not throwing away any information, will produce a very coarse visual display that may lead to misinterpretation of the data. Some scaled spectrograms from oudoors scene are shown in Figure 3.3.

The intensity values in the spectrogram are represented by 256 gray scale values. Most of the pixels in the spectrogram image are produced due to noise, only the very high values gray scale values in the spectrogram image correspond to any significant events in the audio. To filter out the noise we threshold the image with a reletively high value (Figure 3.4). 

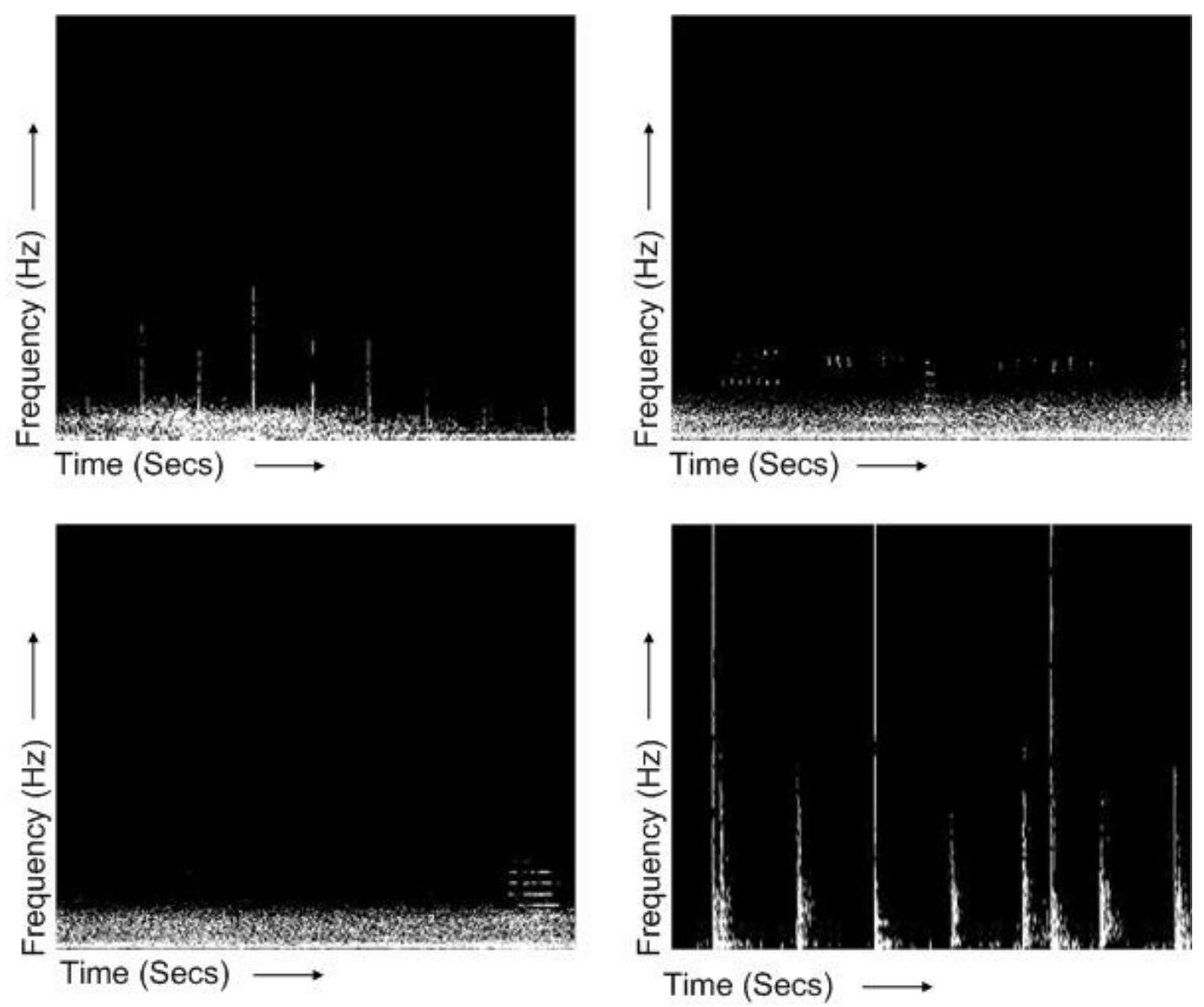

Figure 3.4 Thresholded Spectrograms (Top Left) Footsteps and Moving Car (Top Right) Sound of Chirping Bird and Car Horn (Bottom Left) Car Horn (Bottom Right) Bouncing Ball and Footsteps

\subsubsection{Line Detection}

A common observation from inspection of several spectrograms is that significant audio events such as a walking person, bouncing ball, car horn etc. produce straight lines in the spectrogram. In this thesis Steger's line detection [19] has been used to extract these straight lines. Steger's line detection proceeds in four stages. First, isolated line points are extracted. These points are characterized as having a zero crossing in the first directional derivative in the direction where the second directional derivative attains its maximum absolute value. Therefore, the algorithm needs to calculate the partial derivatives of the image. This is done by convolving it with the partial derivatives of a Gaussian smoothing kernel with scale $\sigma$. The result of this step are individual line points and their subpixel locations, as well as the direc- 

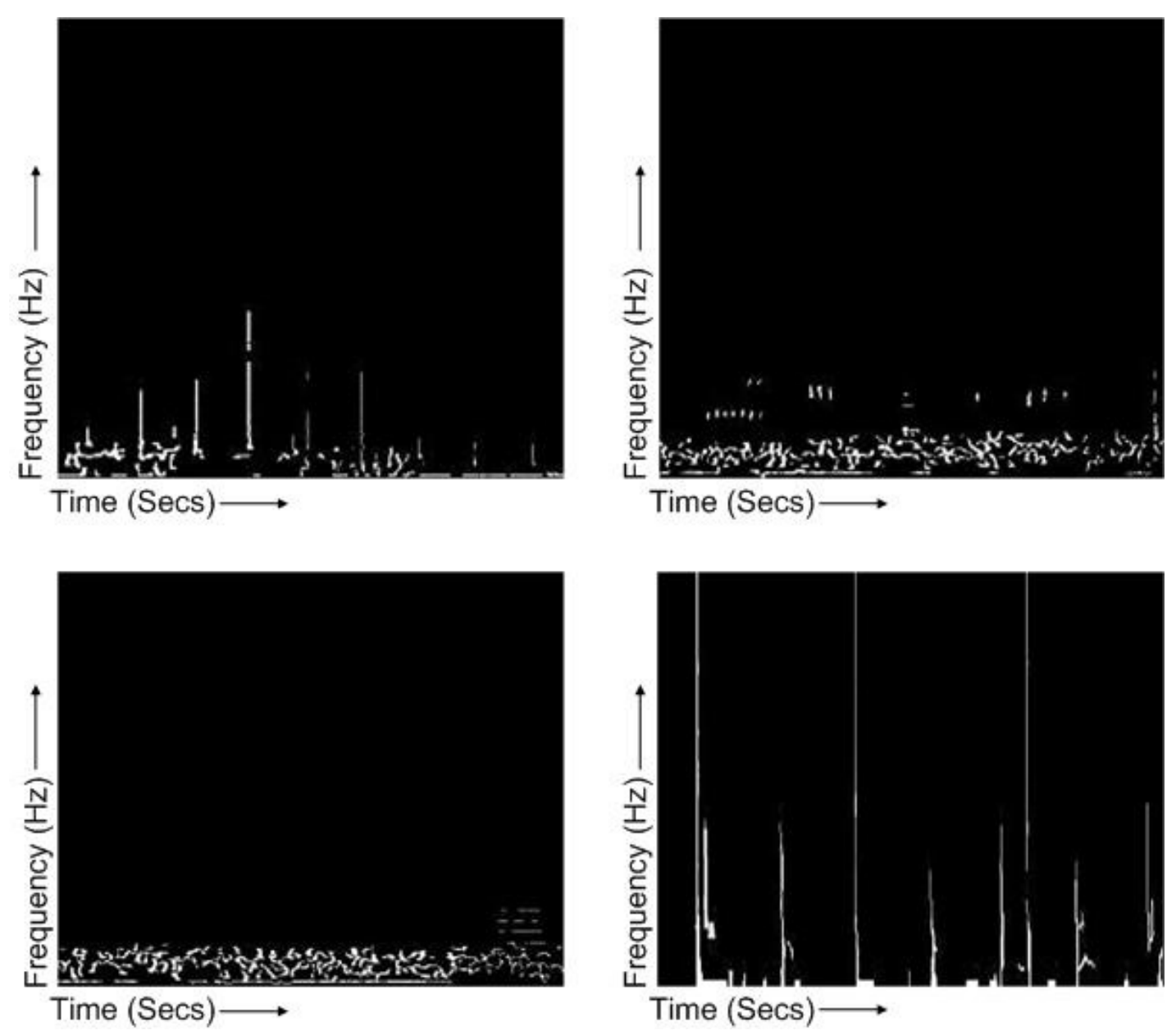

Figure 3.5 Spectrogram Images with Detected Lines (Top Left) Footsteps and Moving Car (Top Right) Chirping Bird and Car Horn (Bottom Left) Car Horn (Bottom Right) Bouncing Ball and Footsteps

tions perpendicular to the line. In the second stage of the algorithm the individual line points are linked into lines. The algorithm starts at points that have a second directional derivative larger than a chosen high value, and follows lines until the second directional derivative is smaller than a low. This procedure is known as a hysteresis threshold operation. The third stage of the algorithm extracts the width of the line for each line point. The line width is extracted by searching for points that have a maximum in the absolute value of the gradient in the direction given by the normal to the line. Only pixels lying on a search line normal to the current line point are considered. The length of the search line is $2.5^{*} \sigma$ on each side of the line. The final stage of the algorithm is to correct the line position and width. Some spectrogram images after line detection have been shown in Figure 3.5. 
Table 3.1 Orientation Groups

\begin{tabular}{|c|c|}
\hline Group & Orientation \\
\hline 1 & $0-30$ and $150-180$ \\
\hline 2 & $30-60$ \\
\hline 3 & $60-120$ \\
\hline 4 & $120-150$ \\
\hline
\end{tabular}

\subsubsection{Grouping}

Significant audio events generally produce parallel lines in the spectrogram. Now our task is to group lines which belong to a specific event. Once we obtain the spectrogram image with the lines representing only the significant audio events, the orientation and centroid of each line is calculated. The orientation is the angle between the $x$-axis and the major axis of the ellipse that has the same second moments as the line. The centroid is the center of mass of the region. The lines are then grouped based on orientation values as shown in Table 3.1. Though this step will group all lines with similar orientation, lines with different pitch information may also be grouped together. To avoid this we further group lines in each orientation group based on the centroid. The co-ordinates of the centroids of lines are clustered using a simple k-means algorithm. This location based grouping extracts lines that are close together and along the same co-ordinate axis signifying lines belonging to the same auditory event. This process however still results in groups which are formed due to low frequency noise in the spectrogram. These groups are ignored during the association stage where they do not find any video object to associate themselves to. Some of the audio groups are shown in Figure 3.6.

\subsubsection{Property Extraction}

Once we isolate the auditory events from the spectrogram it is easy to estimate its periodicity. First we find the column-wise summation of each auditory group as shown in 3.7. The peaks in the auditory signal can be obtained by finding the local maximas in the column summation array of the audio group. By finding the corresponding column of each local maxima we can find the column difference between each peak by subtracting consecutive peak column numbers. This can be converted to time by using: 


$$
\text { Time Between Peaks }=\frac{\text { Column Difference }}{\text { Total Number Of Colums }} \times \text { Total time }
$$

The average of the time difference between each local maxima gives the average periodicity of the auditory object.

\subsection{Grouping Video Objects}

Moving objects can be detected by background subtraction. Once we subtract background frame from each frame we threshold the image to get a binary version with just the objects in motion. Video objects exhibit two types of periodicities: shape change periodicity or track periodicity. Periodic behavior of objects such as humans exhibit shape change periodicity and can be estimated by measuring the change in the number of pixels in the bounding box in each frame. This is based on the fact that in a $2 \mathrm{D}$ image the number of pixels corresponding to the human decrease as both the legs come together and increase as the step is completed (Figure 3.8). The periodic behavior of objects such as a bouncing ball can be calculated by tracking the centroid of the bounding box in each frame (Figure 3.9). Once we have the periodic curves we can find the periodicity by applying periodic transforms [20].

The Periodicity Transform searches for the best periodic characterization of the length $N$ signal $x$. The underlying technique is to project $x$ onto some periodic subspace $S p$. This periodicity is then removed from $x$ leaving the residual $r$ stripped of its p-periodicities. Both the projection $x$ and the residual $r$ may contain other periodicities, and so may be decomposed into other $q$-periodic components by further projection onto $S q$. This decomposition is accomplished directly in terms of periodic sequences and not in terms of frequency or scale, as do the Fourier and Wavelet Transforms. Unlike most transforms, the set of basis vectors is not specified a priori, rather, the Periodicity Transform finds its own best set of basis elements.

Though we estimate both the periodicities for each object, only one type of periodicity will be relevant to an object. For example, the human might exhibit periodic motion by monitoring the change in pixels in a bounding box, but tracking the centroid will reveal only 
a straight line. The periodic transform will estimate periodicity as zero and hence we can ignore it. The periodicity estimate in terms of number of frames can be converted to time by:

$$
\text { Video Periodicity }=\frac{\text { Number Of Frames }}{\text { Total Number Of Frames }} \times \text { Total time }
$$

\subsection{Association}

Audio and video data are highly correlated, for example the sound of a ball bounce will be accompanied by an event in the video where the ball comes in contact with the floor (Frames 21, 52, 83, 116 Figure 3.10). Similarly, the sound of a footstep will be accompanied by the foot of a person hitting the floor (Frames 36, 50, 63, 91, 106, 118, 131 in Figure 3.10). We simply need to keep track of when we hear the sound and in video we need keep track of when the ball or the foot comes in contact with the floor. Essentially, the periodicities of objects both in audio and video should be similar. Video will have exactly the same number of periodicities as the number of objects in the scene. However, in audio due to noise, our procedure might pick up false periodicities. To eliminate these we simply do a percentage difference check between the video and audio periodicities. The lowest differences will give us the video object and audio object which are most similar.

$$
\text { Difference Percentage }=\frac{\text { Audio Periodicity }- \text { Video Periodicity }}{\text { Audio Periodicity }} \%
$$



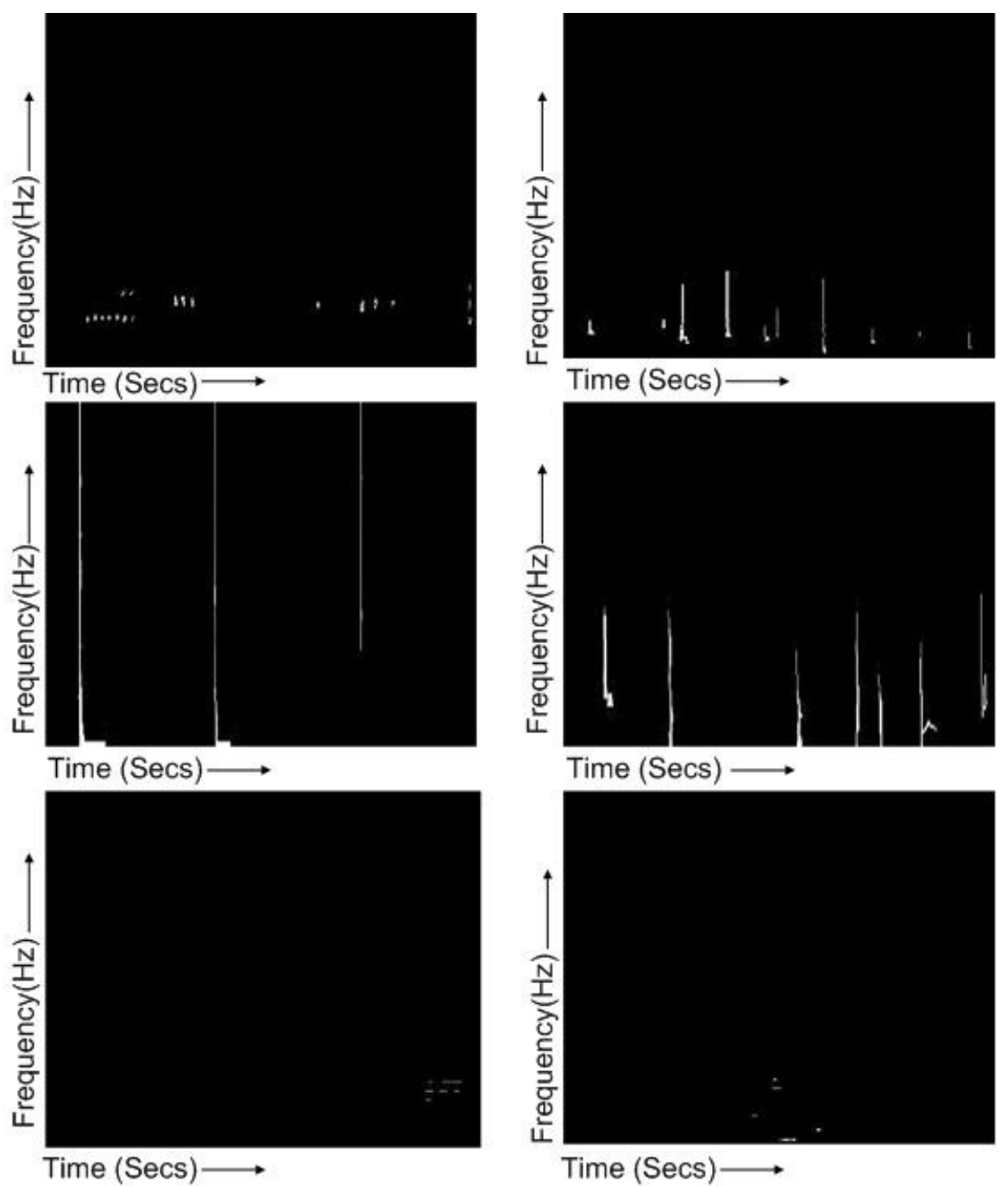

Figure 3.6 Isolated Auditory Events (Top Left) Bird Chirping (Top Right) Footsteps (Middle Left) Ball Bounce (Middle Right) Footsteps (Bottom Left) Car Horn (Bottom Right) Truck Horn 


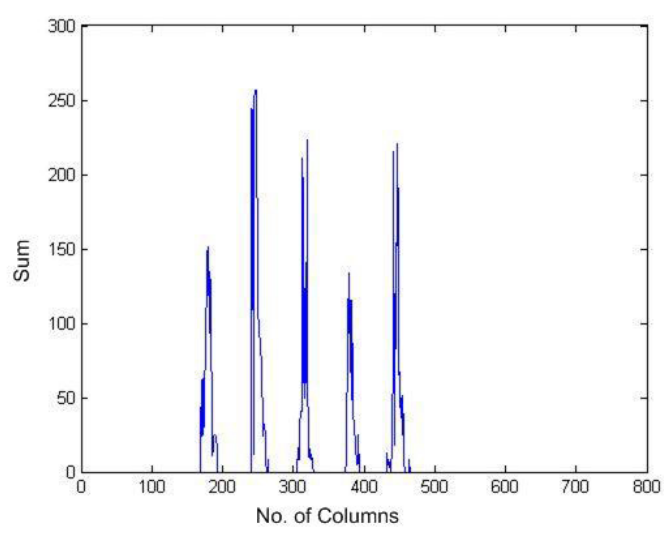

Figure 3.7 Column Wise Summation of an Auditory Group
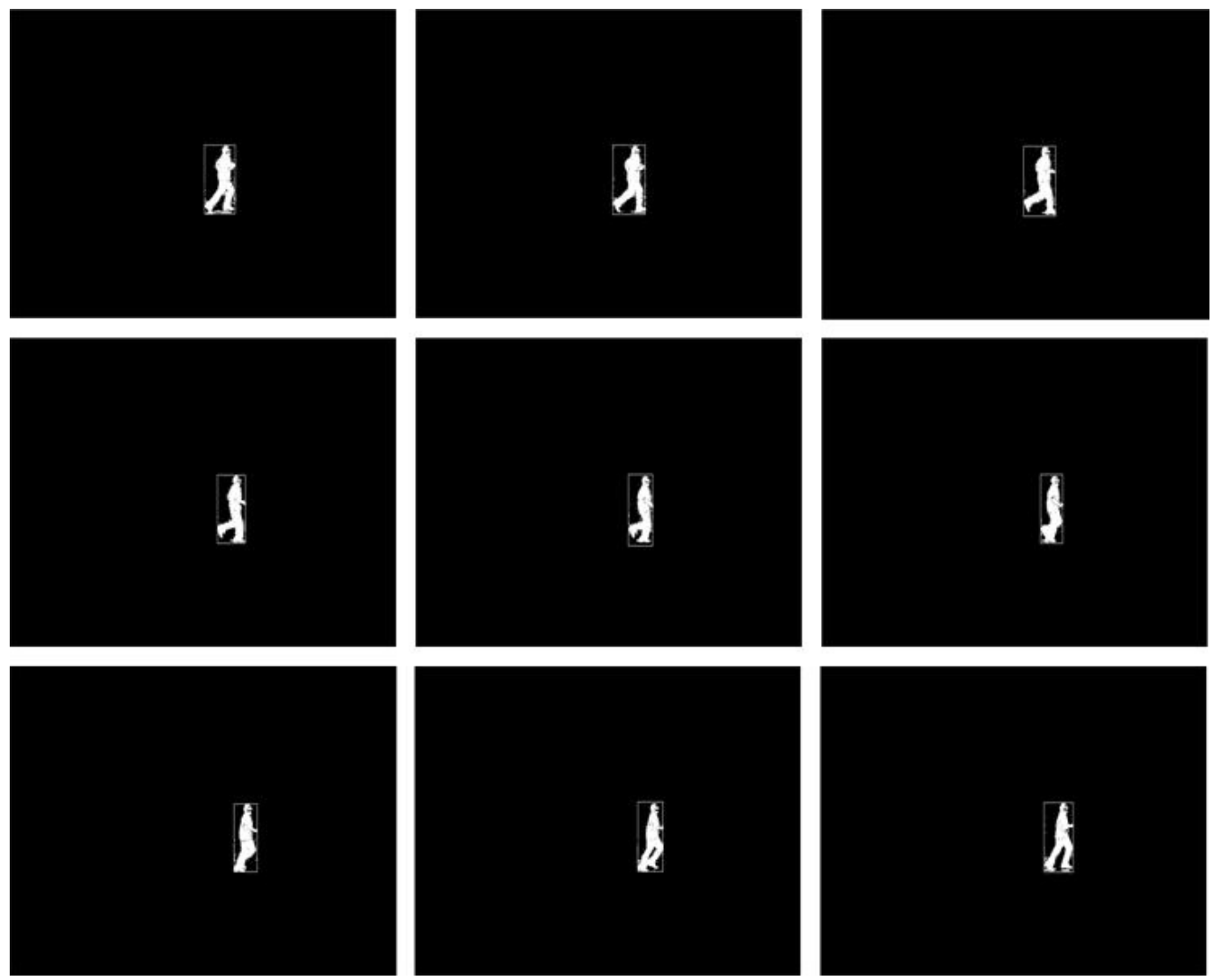

Figure 3.8 A Walking Person Exhibits Shape Change Periodicity 


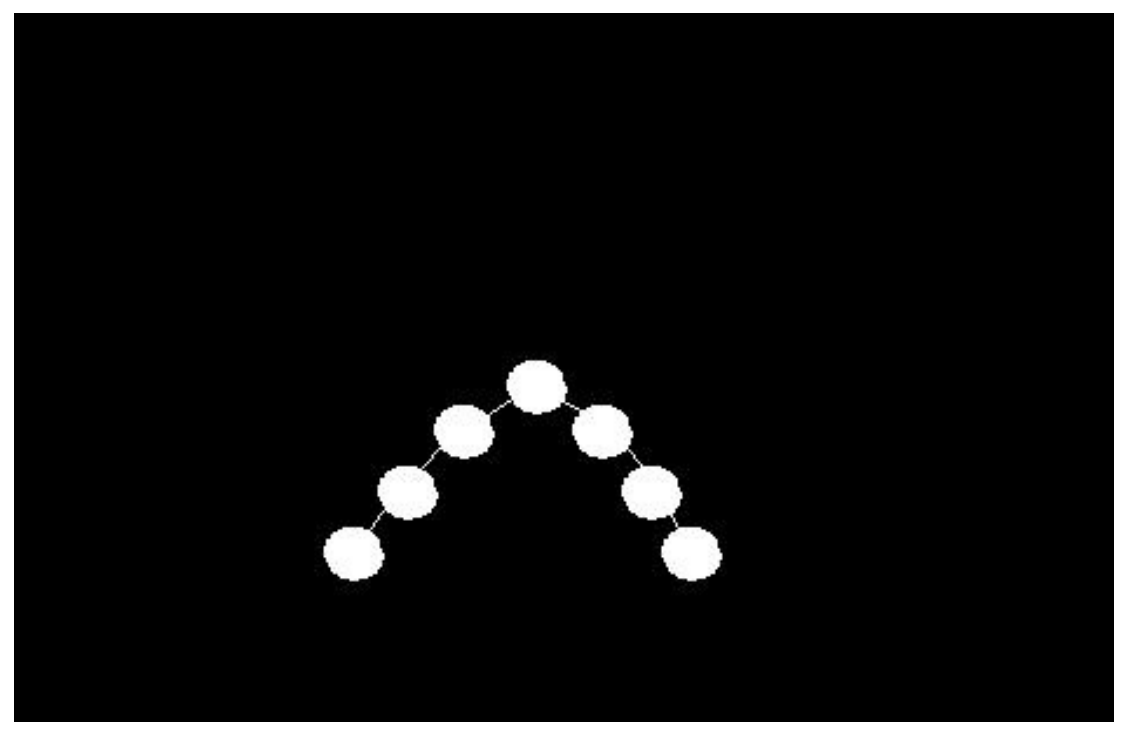

Figure 3.9 A Bouncing Ball Exhibits Track Change Periodicity

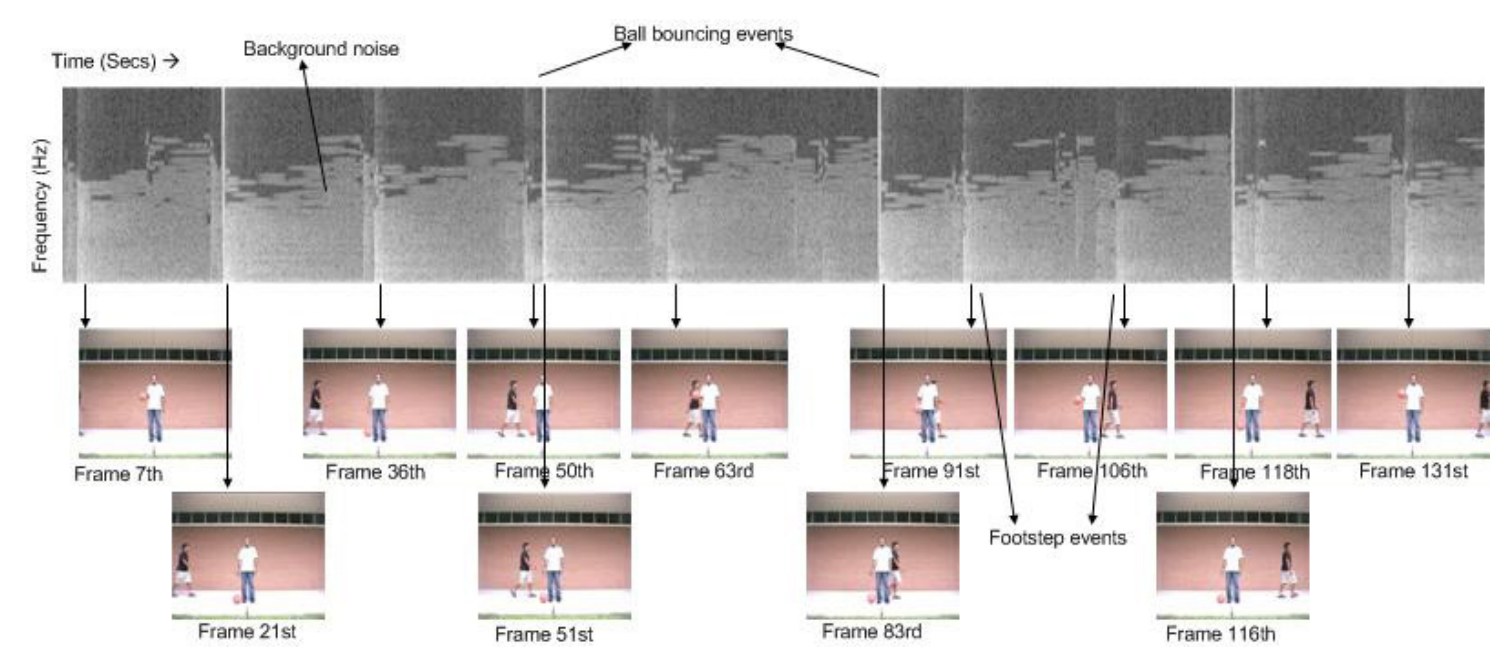

Figure 3.10 (Top) Spectrogram of the Sound Waveform Received. (Bottom) Corresponding Frames for Each Event in the Spectrogram 


\section{CHAPTER 4}

\section{RESULTS}

In this chapter we consider certain scenarios consisting of objects that exhibit periodic motion. The audio and video results of each scenario have been shown in this thesis as well as on the CD provided with this report.

\subsection{Scenario 1: Person Bouncing Ball and Walking Person}

First, we consider a small video clip showing a person bouncing a ball and a person walking (CLIP 1 on CD). The corresponding spectrogram of the audio signal, the thresholded spectrogram and the detected lines are generated as described is the previous chapter (Figure 4.1). The auditory groups formed are shown in Figure 4.2. At this point we get three objects, though in reality there are only two sound producing objects and the other is auditory object is formed due to noise. Using local maxima to detect peaka in audio signal, the periodicities of the audio groups shown are estimated to be 1.06, 0.8 and 0.46 seconds respectively.

Some of the video frames are shown in the top section of Figure 4.3. Motion segmentation gives us two objects. Each object either gives us shape periodicity or track periodicty. How-
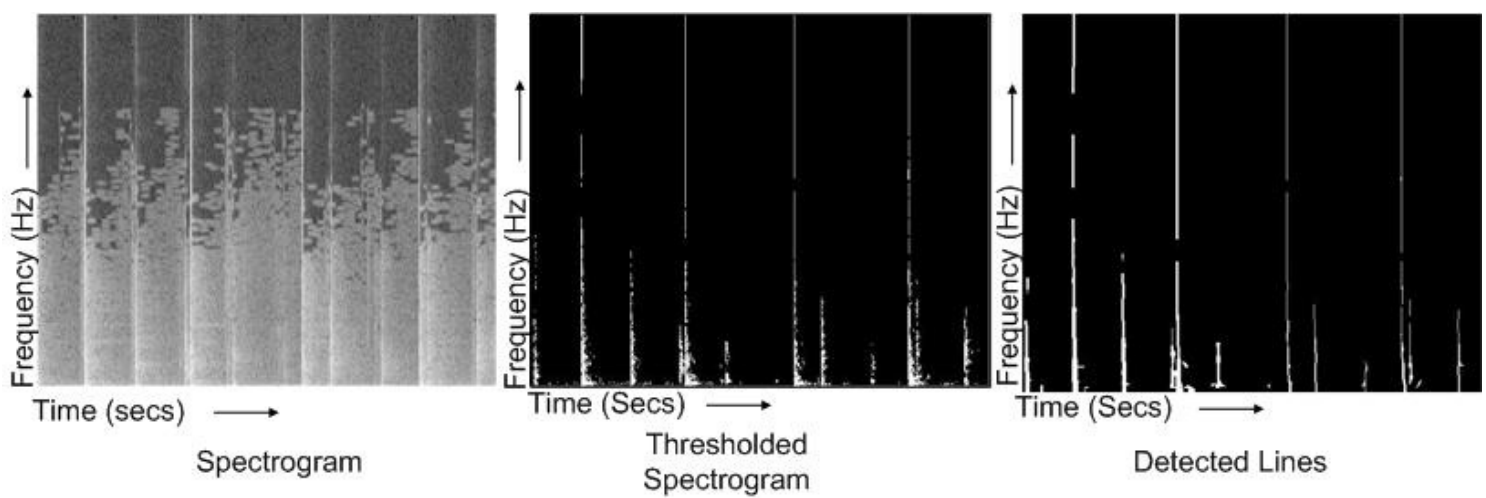

Figure 4.1 (Left) Spectrogram (Middle) Thresholded Spectrogram (Right) Detected Lines 

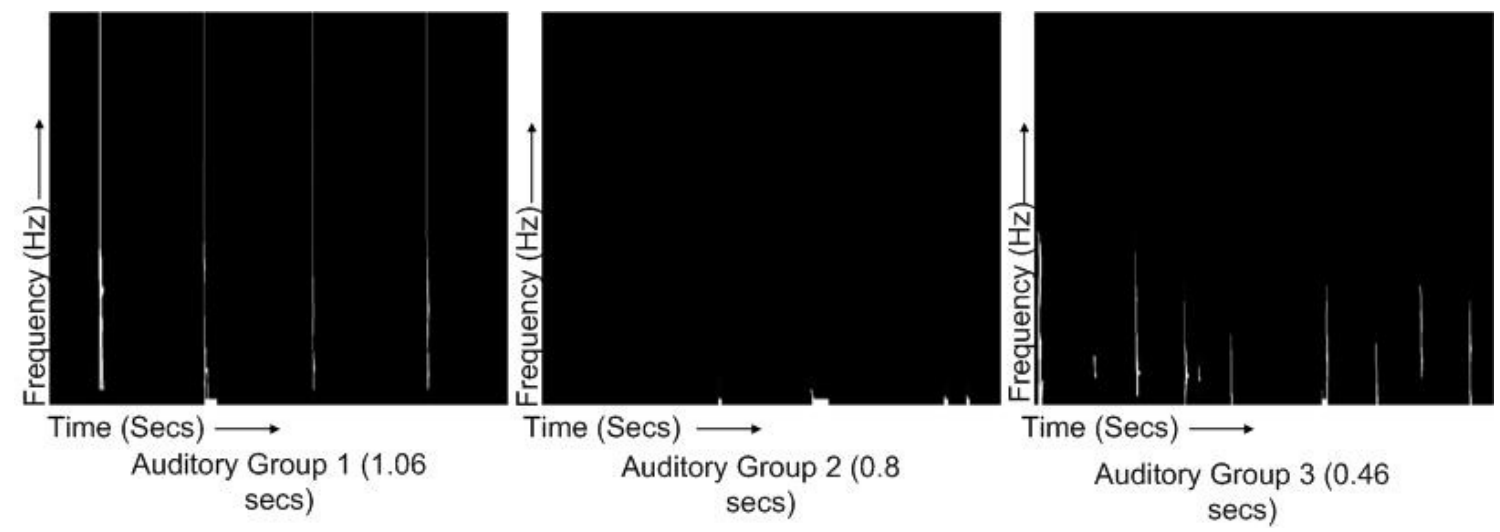

Figure 4.2 Audio Groups

Table 4.1 Difference Percentages: Video Object 1=Bouncing Ball Video Object 2=Footsteps

\begin{tabular}{|c|c|c|}
\hline Audio Object number (Periodicity) & Video Object number(Periodicity) & Difference \% \\
\hline 1 (1.06 seconds) & $1(1.04$ seconds $)$ & $0.02 \%$ \\
\hline $2(0.8$ seconds $)$ & $1(1.04$ seconds $)$ & $0.30 \%$ \\
\hline $3(0.46$ seconds $)$ & $1(1.04$ seconds $)$ & $1.26 \%$ \\
\hline $1(1.06$ seconds $)$ & $2(0.52$ seconds $)$ & $0.51 \%$ \\
\hline $2(0.8$ seconds $)$ & $2(0.52$ seconds $)$ & $0.35 \%$ \\
\hline $3(0.46$ seconds $)$ & $2(0.52$ seconds $)$ & $0.13 \%$ \\
\hline
\end{tabular}

ever, the type of periodicty is irrelevant for association. The relevant periodicity curves are in the bottom section of Figure 4.3. Once we apply periodicity transform we get periodicities 1.04 seconds and 0.52 seconds respectively.

Once we obtain the auditory and visual periodicities, we calculate the difference percentages (Table 4.1). Since we have two video objects, the smallest two percentages form the association. Hence, from the table the first association is formed between visual object 1 and audio object 1 and the second association is formed between visual object 2 and audio object 3. Visually, the bouncing ball video object is associated with audio object three and human walking is associated with audio object one as shown in Fig. 4.4.

Spectrogram images of the isolated auditory events can also be used to regenerate the corresponding audio signal. By retaining only the columns in which atleast one pixel is equal to one, in the original spectrogram matrix (obtained by applying Fourier transform to the original wave form) and making all others zero, we can then apply a frame by frame inverse Fourier transform and append the signal to obtain the corresponding sound [21]. We can 

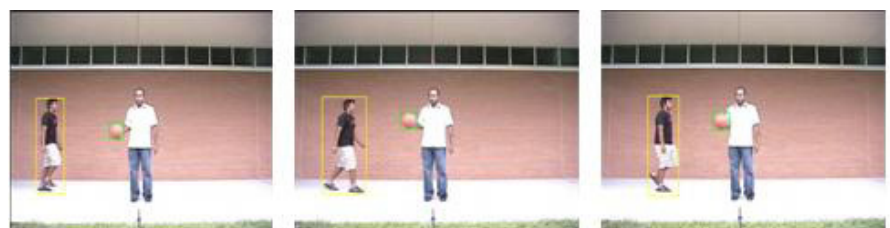

Video frames
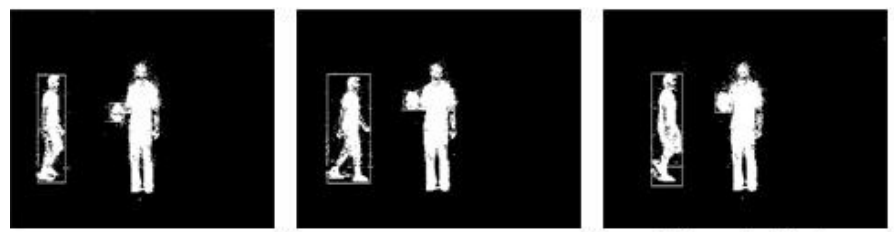

Thresholded frames
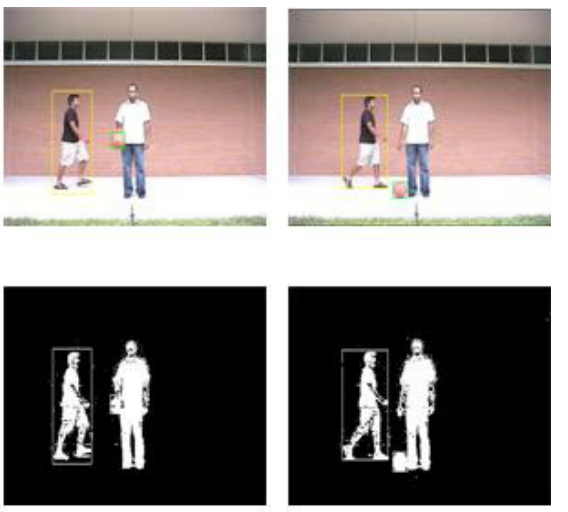

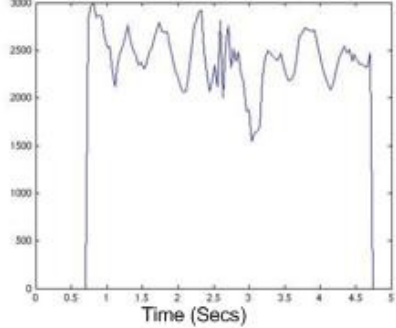

Periodicity curve of the walking person

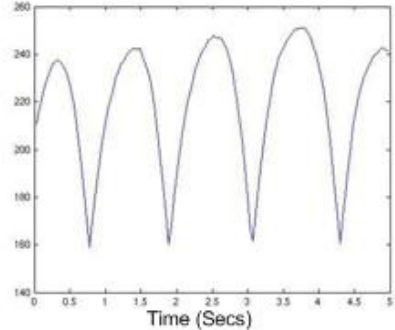

Periodicity curve of the bouncing ball

Figure 4.3 (Top) Video Frames (Middle) Thresholded Frames (Bottom) Periodicity Curves

regenerate the sound corresponding to the relevant auditory object. CLIP 2 and CLIP 3 on the CD exhibit the audio-video association. Follow the yellow box in each clip and hear the sound which has been associated to it.

\subsection{Scenario 2: Walking Person and Moving Car}

Now, we consider another scenario in which we have a walking person and car passing by in the background (CLIP 4 on CD). The corresponding spectrogram of the audio signal and some of the auditory groups formed are shown in Fig. 4.5. We expect to find one audio object for the footsteps and another for the sound of the car. However, the sound of a car creates a noise like signature in spectrogram and hence the line detection cannot pick up the sound of the car. In this case, one audio object corresponding to the persons footsteps and other is formed due to noise. The periodicities of the audio groups shown are 0.53 and 1.54 seconds respectively. Some of the video frames are shown in the top section of Fig. 4.6. The periodicity curves 


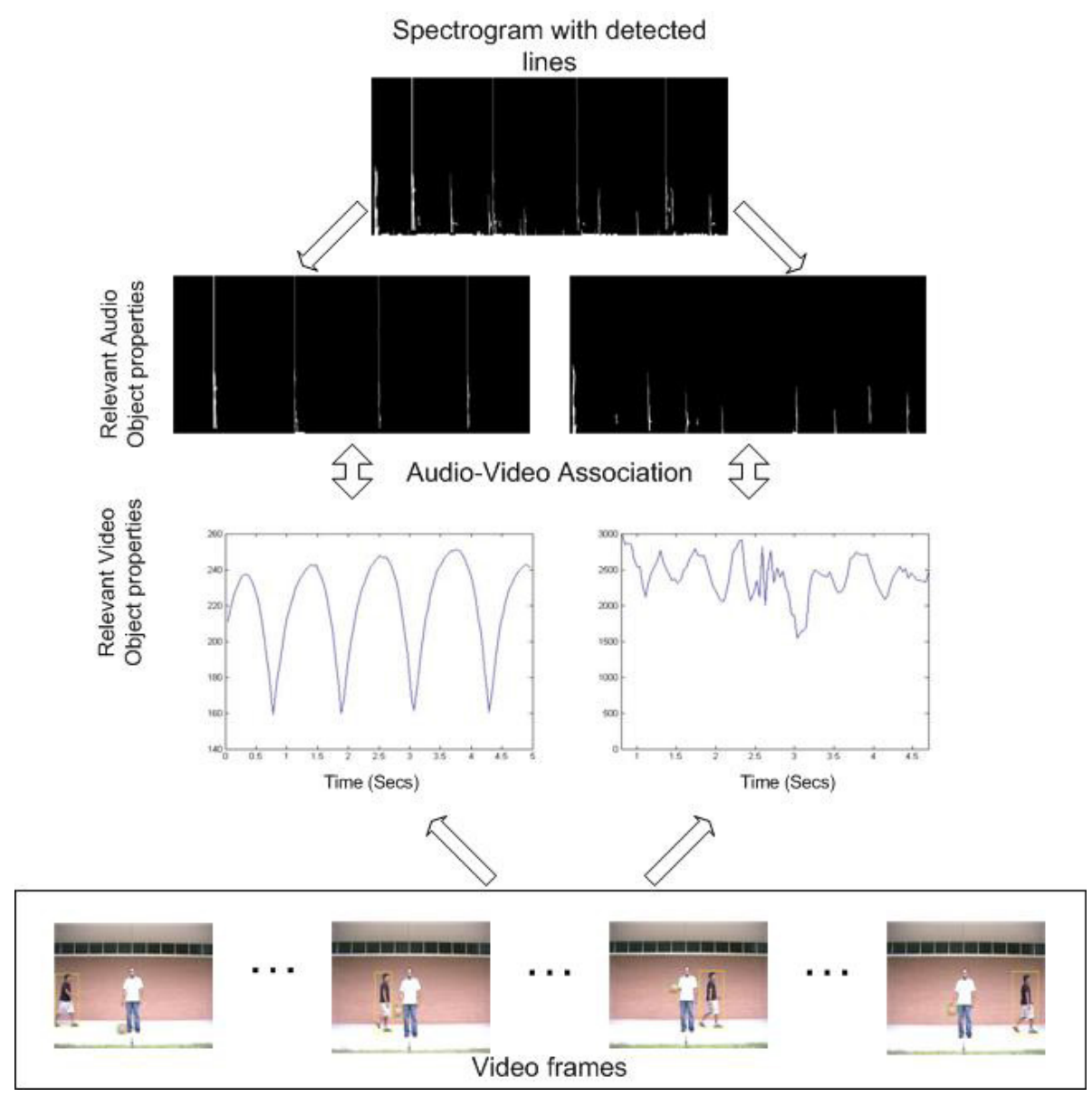

Figure 4.4 Association of Sound - Top Row Shows the Lines Detected from the Spectrogram of the Sound Wave from Which We Obtain Audio Objects Shown in the Second Row. Third Row Shows the Periodicity Curves of Objects in the Video Extracted from Video Frames Shown in the Last Row

are in the bottom section of Fig. 4.6. The walking person exhibits shape periodicty, however the moving car does not exhibit any periodicity. Once we apply periodicity transform we get periodicities 0.55 and zero seconds respectively. Association relates audio object one to the walking person (CLIP 5 on CD) but no audio object is associated to the moving car. We indicate this in Fig. 4.7 by not showing any bi-directional arrow for the second video object.

\subsection{Scenario 3: Person Walking and Another Person Running}

Now, we consider a scenario in which we have a walking person and another person running (CLIP 6 on CD). The corresponding spectrogram of the audio signal, the detected lines and 

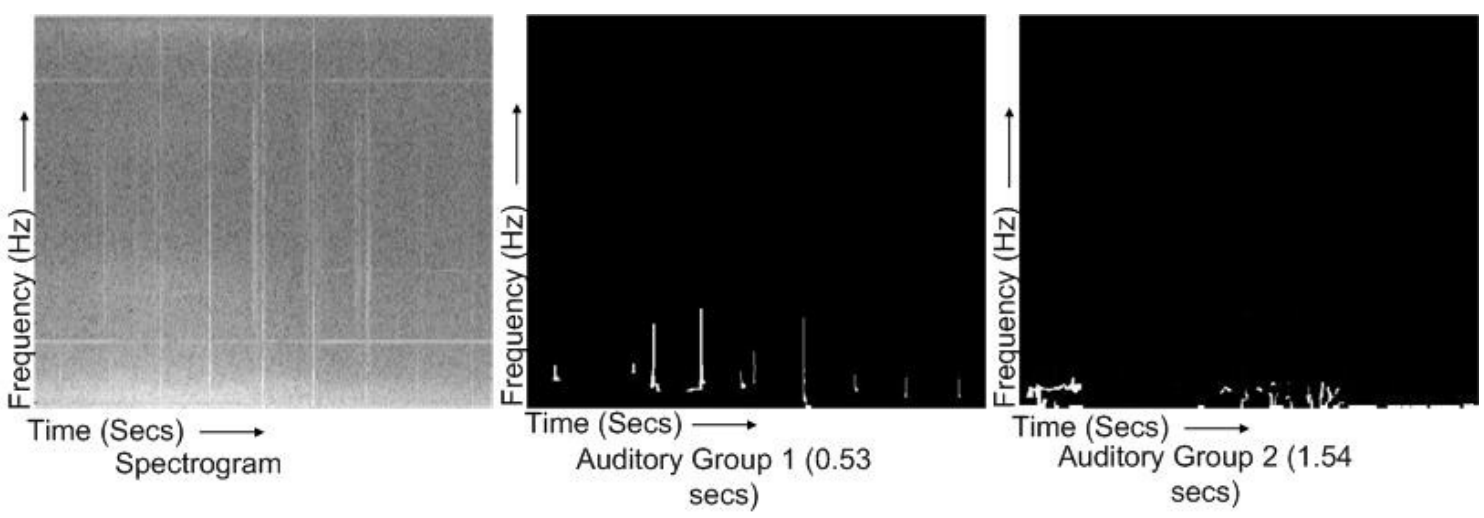

Figure 4.5 Spectrogram and the Corresponding Audio Groups

some of the auditory groups formed are shown in the top 3 rows of Fig. 4.8. The periodicities of the audio groups shown are $0.23,0.5$ and 0.4 seconds respectively. Some of the video frames are shown in the bottom row of Fig. 4.8. The periodicity curves are in the forth row of Fig. 4.8 .

Both video object exhibit shape periodicities. Once we apply periodicity transform we get periodicities 0.52 and 0.41 seconds respectively. The first audio object is formed due to noise. Association relates second audio object to the running person (CLIP 7 on CD). The third corresponds to the footsteps of the walking person (CLIP 8 on CD).

\subsection{Scenario 4: Person Walking and Bouncing Ball}

Now, we consider a scenario in which we have a walking person and a bouncing ball (CLIP 9 on $\mathrm{CD}$ ). The corresponding spectrogram of the audio signal, the detected lines and some of the auditory groups formed are shown in the top 3 rows of Fig. 4.9. The periodicities of the audio groups shown are 0.38 and 0.73 seconds respectively. Some of the video frames are shown in the bottom row of Fig. 4.9. The periodicity curves are in the forth row of Fig. 4.9. Once we apply periodicity transform we get periodicities 0.4 and 0.7 seconds respectively. A percentage difference check corresponds first audio object to the footsteps of the running person (CLIP 11 on CD) and the second corresponds to the bouncing ball(CLIP 10 on CD). 

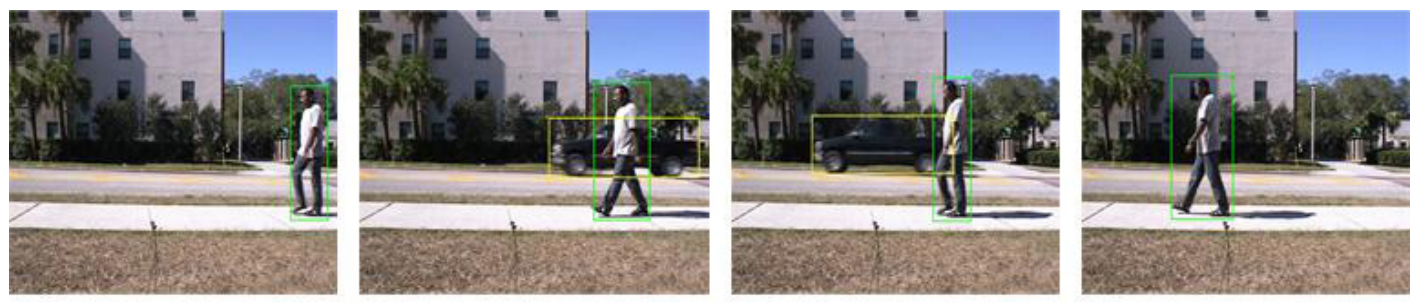

Video frames

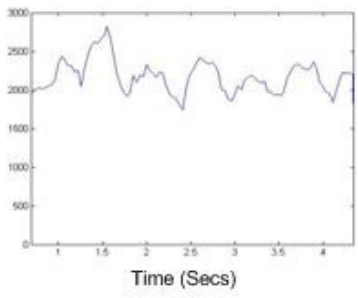

Periodicity Curve of

Walking Person

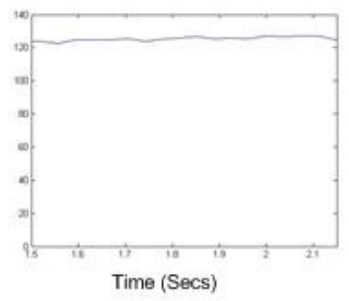

Periodicity Curve of Moving Car

Figure 4.6 (Top) Video Frames (Bottom) Periodicity Curves

\subsection{Scenario 5: Person Walking, Person Running and Bouncing Ball}

Now, we consider another scenario in which we have a walking person, a running person and a bouncing ball (CLIP 12 on CD). The corresponding spectrogram of the audio signal, the detected lines and some of the auditory groups formed are shown in the top 2 rows of Fig. 4.9. The periodicities of the audio groups shown are $0.61,1.1$ and 0.22 seconds respectively. Some of the video frames are shown in the bottom row of Fig. 4.9. The periodicity curves are in the forth row of Fig. 4.9. Once we apply periodicity transform we get periodicities $0.6,1.1$ and 0.23 seconds respectively. The first corresponds to the footsteps of the running person (CLIP 13 on CD). The second corresponds to the bouncing ball (CLIP 14 on CD) and third corresponds to walking person (CLIP 15 on CD). 

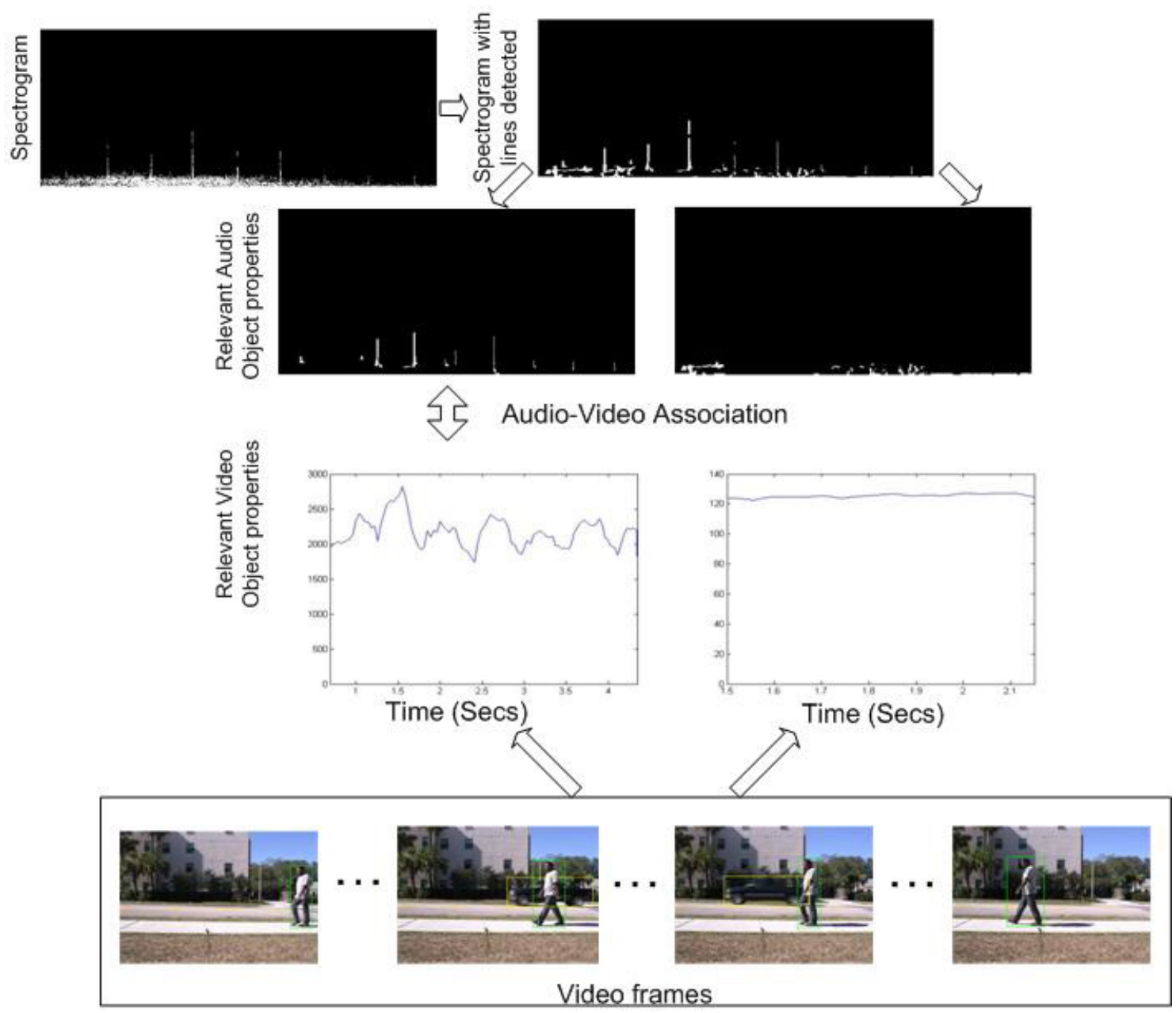

Figure 4.7 Top Row Shows the Spectrogram on the Left Side and Lines Detected on the Right Side. Two of the Audio Objects are Shown in the Third Row. Fourth Row Shows the Periodicity Curves of Objects in the Video Extracted from Video Frames Shown in the Last Row 


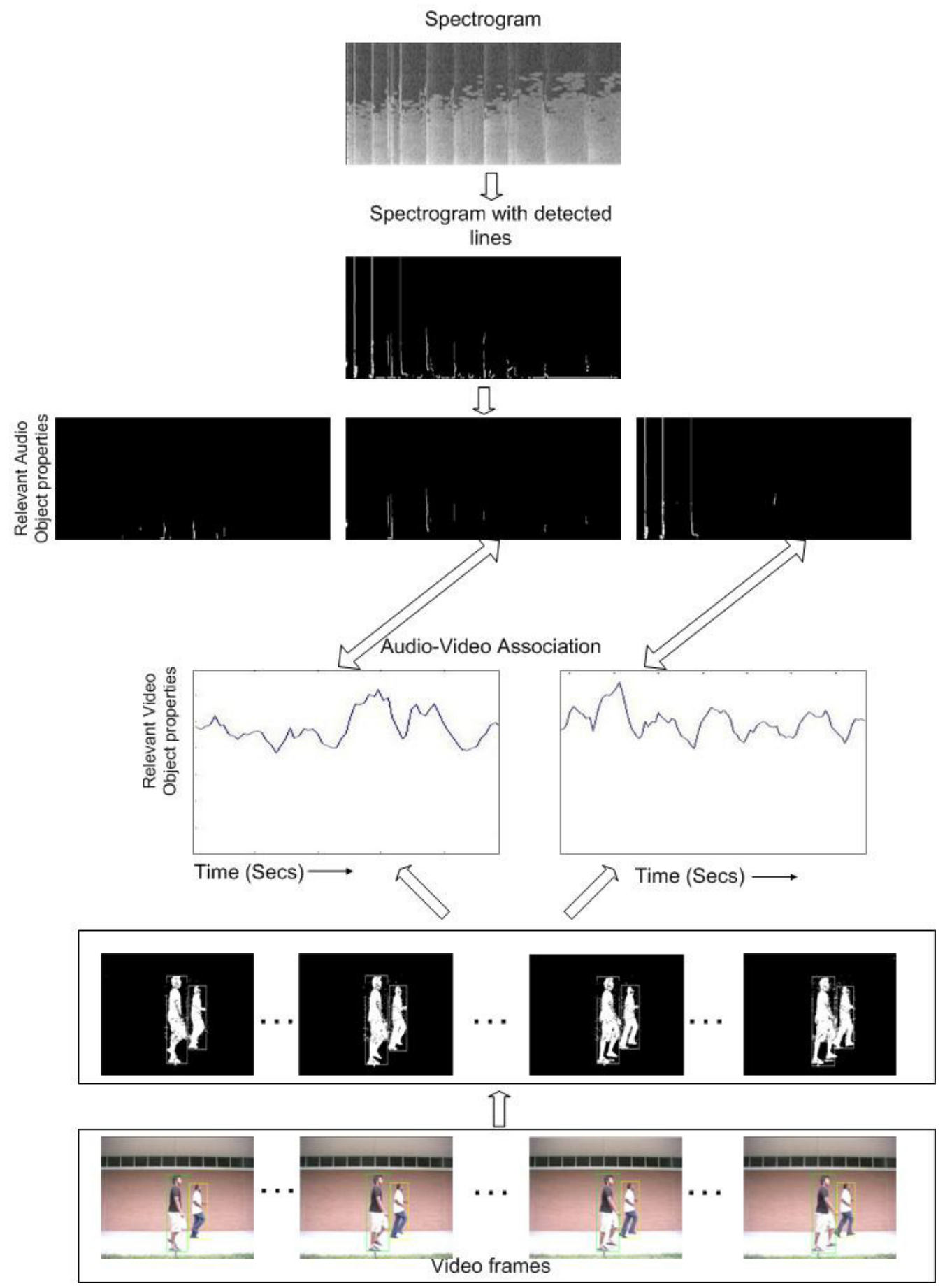

Figure 4.8 Top Three Rows Show the Spectrogram, Detected Lines and the Audio Objects. Fourth Row Shows the Periodicity Curves of Objects in the Video. The Fifth Row Shows Thresholded Frames and the Last Row Shows Video Frames 


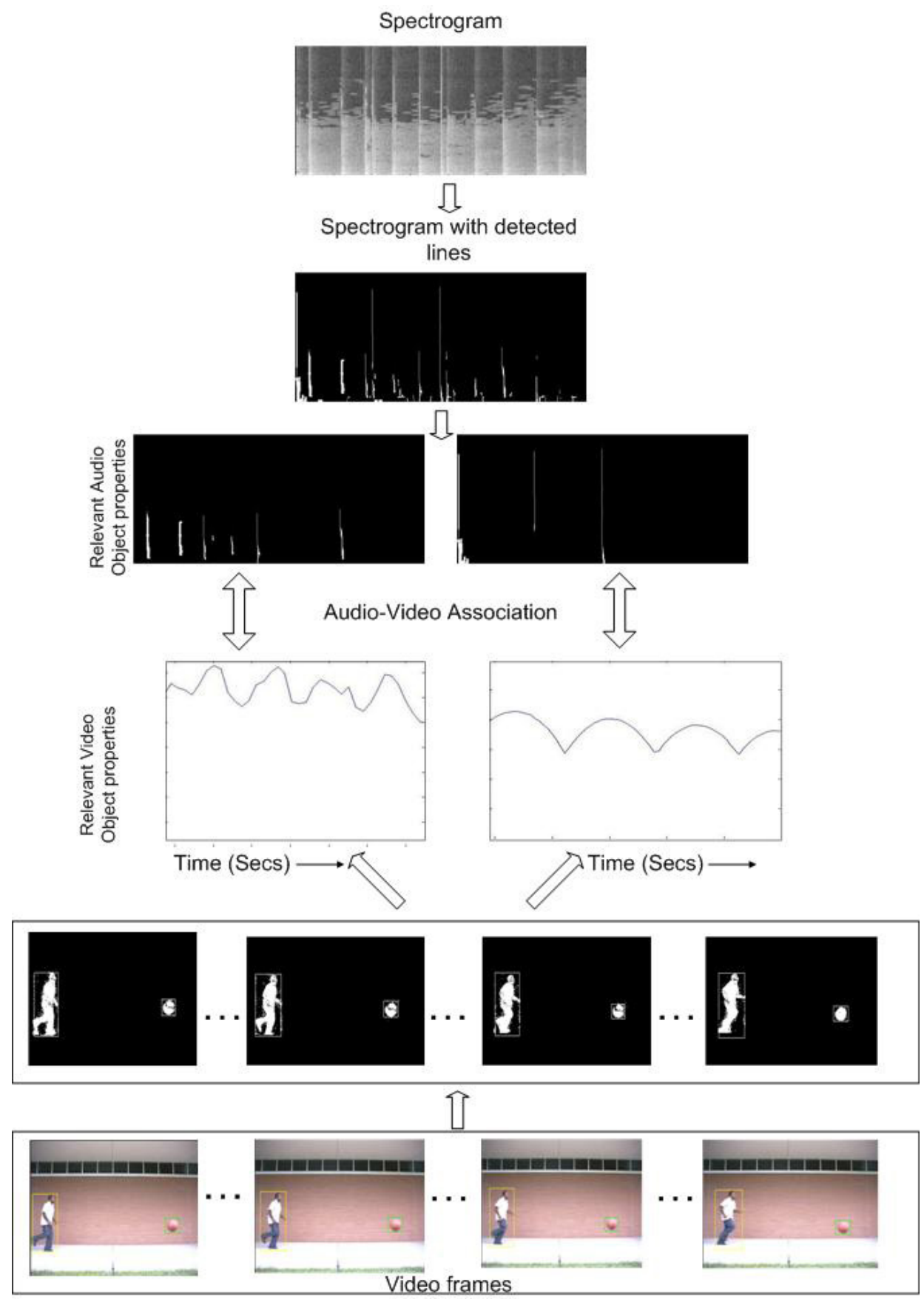

Figure 4.9 Top Row Shows the Spectrogram and the Next Row Shows the Lines Detected. Two of the Audio Objects are Shown in the Third Row. Fourth Row Shows the Periodicity Curves of Objects in the Video Extracted from Video Frames Shown in the Last Row 


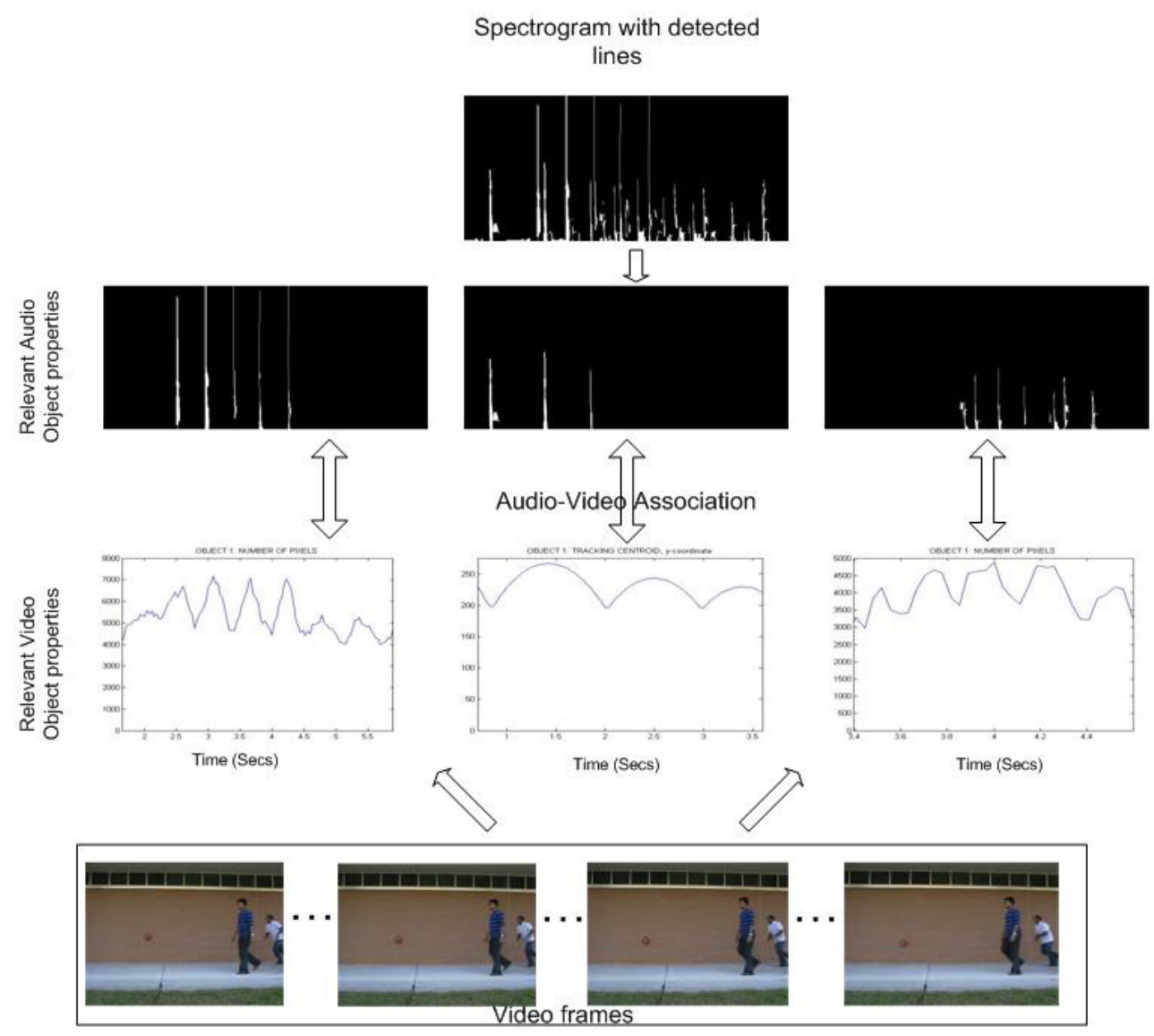

Figure 4.10 Top Row Shows the Lines from the Spectrogram. Three of the Audio Objects are Shown in the Second Row. Third Row Shows the Periodicity Curves of Objects in the Video Extracted from Video Frames Shown in the Last Row 


\section{CHAPTER 5 \\ CONCLUSIONS}

In this thesis, we present a technique by which we can associate sound to motion in the video. Specifically, we associate sound to objects exhibiting shape change or track periodicities. Our method uses a feature based approach that groups high level primitives, thus avoiding the noise associated with using low level features. This approach works well even in a cluttered environment and can associate more than one sound to the respective objects at the same instant. Contrary to the traditional signal processing techniques, we use auditory scene analysis techniques to process audio. This gives us separate audio events present in the sound signal that are then grouped based on periodicity of the audio signal. Unlike many well-known signal processing techniques which use multiple microphones to collect audio data, we stick to audio data received by a single microphone.

In an age when video surveillance has become utmost important, association of sound to video can help zoom in on interesting objects in the video. It can also help figure out if the sound produced is within the visual range. The camera can then pan to see if it can find any object of interest.

Though this technique uses primarily periodicity as its cue to form audio-video association, we believe the concurrent nature of the audio-video data exhibit many more high level primitives which can be used for association. Multi sensor processing finds its use in many areas including video surveillance. Psychological evidence and analysis of biological systems has shown that audio-visual fusion enhances perception. 


\section{REFERENCES}

[1] I A Essa. Ubiquitous sensing for smart and aware environments: technology towards the building of an aware home. In IEEE Personal Communications, pages 47-49, 2000.

[2] A S Bergman. Auditory scene analysis: The perceptual organisation of sound. MIT Press, 1990.

[3] H McGurk and J MacDonald. Hearing lips and seeing voices. Nature, pages 746-748, 1976.

[4] J Vroomen, P Bertelson and B de Gelder. A visual influence in the discrimination of auditory location. In Proceedings of the International Conference on Auditory-Visual Speech processing, pages 131-135, 1998.

[5] J Vroomen and B de Gelder. Sound enhances visual perception: Cross-Modal effects of auditory organization on vision. Journal of Experimental Psycology: Human perception and performance, pages 1583-1590, 2000.

[6] G Mather. The motion after effect: A modern perspective. MIT Press, 1998.

[7] R A Berman and C L Colby. Auditory and visual attention modulate motion processing in area MT+. Cognitive Brain Research, 14:64-74, 2002.

[8] D Lo and R A Goubran . Auditory and visual attention modulate motion processing in area MT+. In IEEE Transactions on instruments and measurements, volume 20, pages 1132-1139, 2004.

[9] D Johnson and D Dudgeon. Array Signal Processing, pages 112-113. Prentice-Hall, 1993.

[10] E Menegatti, E Mumlo, M Nolich and E Pagello. A surveillance system based on audio and video sensory agents cooperating with a mobile robot. In International Conference on Intelligent Autonomous Systems, pages 335-343, 2004.

[11] S Tsekeridou and I Pitas. Content-Based video parsing and indexing based on audiovisual interaction. In IEEE Transactions on circuits and systems for video technology, volume 11, pages 522-535, 2001.

[12] R Cutler and L Davis. Look who's talking: Speaker detection using video and audio correlation. In IEEE International Conference on Multimedia and Expo, volume 3, pages 1589-1592, 2000.

[13] E Kidron, Y Y Schechner and M Elad. Pixels that Sound. In International conference on pattern recognition, volume 1, pages 88-95, 2005. 
[14] L McPherson, V Ciocca and A Bergman. Organization in audition by similarity in rate of change: evidence from tracking individual frequency glides in mixtures. Perception and Psychophysics, pages 269-278, 1994.

[15] G Kanizsa. Organization in vision: essays on Gestalt principle. New York:Praeger, 1979.

[16] G Vicario. The acoustic tunnel effect. Rivista da Psicolgia, pages 41-52,54, 1960.

[17] R M Warren. Auditory perception: a new synthesis. New York: Pergamon Press, 1982.

[18] M Kubovy and D V Valkenburg. Auditory and visual objects. Cognition, 80:97-126, 2001.

[19] C Steger. An Unbiased detector of Curvilinear structures. In IEEE Transactions on Pattern Analysis and Machine Intelligence, volume 20, pages 113-135, 1998.

[20] W A Sethares and T W Staley. Periodicity Transforms. In IEEE Transactions on Signal Processing, volume 47, pages 2953-2964, 1999.

[21] D Ellis. Spectrogram invesion. http://www.ee.columbia.edu/ dpwe/resources /matlab/sgram/ispecgram.m. 